NBER WORKING PAPER SERIES

\title{
DURABLE GOODS AND CONFORMITY
}

\author{
Christopher L. House \\ Emre Ozdenoren
}

Working Paper 12028

http://www.nber.org/papers/w12028

\author{
NATIONAL BUREAU OF ECONOMIC RESEARCH \\ 1050 Massachusetts Avenue \\ Cambridge, MA 02138 \\ February 2006
}

The authors gratefully acknowledge the comments of Kai-Uwe Kuhn, Miles Kimball, Greg Lewis, Steve Salant, Dan Silverman and Lones Smith. The views expressed herein are those of the author(s) and do not necessarily reflect the views of the National Bureau of Economic Research.

(C2006 by Christopher L. House and Emre Ozdenoren. All rights reserved. Short sections of text, not to exceed two paragraphs, may be quoted without explicit permission provided that full credit, including () notice, is given to the source. 
Durable Goods and Conformity

Christopher L. House and Emre Ozdenoren

NBER Working Paper No. 12028

February 2006

JEL No.

\begin{abstract}
Is the variety of products supplied in markets a reflection of the diversity of consumers' preferences? In this paper, we argue that the distribution of durable goods offered in markets tends to be compressed relative to the distribution of consumers' underlying preferences. In particular, there are strong incentives for conformity in markets for durable goods. The reason for conformity is natural: durables (for example houses) are traded and as a result, demand for these goods is influenced by their resale value. Agents may like one product, but purchase another because of resale concerns. We show that (1) there is a tendency to conform to the average preference; (2) conformity depends primarily on the number of people with extreme preferences; (3) conformity increases with increases in durability, patience, and the likelihood of trade; and (4) equilibrium conformity is not necessarily optimal. Surprisingly, there tends to be too little conformity in equilibrium. Conformity also creates a demand for rental markets. Renting does not necessarily decrease conformity however. Instead, renting tends to exaggerate conformity in the owner-occupied market.
\end{abstract}

Christopher L. House

University of Michigan

Department of Economics

238 Lorch Hall

Ann Arbor, MI 48109

and NBER

chouse@umich.edu

Emre Ozdenoren

University of Michigan

Department of Economics

238 Lorch Hall

Ann Arbor, MI 48109

emreo@umich.edu 
"Conformity, in terms of size, condition $\&$ features, tends to support your home's market value more than anything else." 1

\section{Introduction}

What determines the mix of products in a market? In standard supply and demand models, the mix of products is determined by preferences and costs. Holding everything else equal, goods which are preferred relative to other goods should be produced and consumed in greater amounts. This prediction rests simply on the idea that consumers demand the goods that they most prefer. On the other hand, people often complain that there is too little variety in certain markets. One market that has received particular attention in this regard is the housing market. New houses often have features and styles that differ only superficially from one house to the next. New housing developments are often derided because they consist merely of "cookie-cutter" houses or "McMansions." These houses are virtually the same - most have cathedral ceilings, walk-in closets, built-in jacuzzis, mud rooms, and so forth. Of course many of these features are desirable but it seems unlikely that preferences are so aligned as to justify such a homogeneous mix of products.

In this paper, we argue that there are powerful incentives for conformity in markets for durable goods. The reason is natural. A durable good, like a house, is traded from time-to-time and as a result, the current demand for the good is influenced by its resale value. Resale concerns can be so strong as to cause an individual to purchase a good that he dislikes relative to other available goods. If this occurs we say that the individual is conforming to the market.

We analyze these issues with a matching model in which agents buy and sell a durable good. The durable good is a long-lived durable that must be resold from time-to-time. Although the specific function of the durable is not important for the analysis, we refer to the durable as a house. There are two types of houses in the market and agents differ according to their preferences over the two types. Frictions in the resale market imply that agents are not matched perfectly with others who have the same preferences. Thus, if someone buys an unusual house, he runs the risk that he will not be able to sell it if he needs to move. We show that in equilibrium, there is a tendency to conform to the average preference. That

\footnotetext{
${ }^{1}$ Quoted from Accurate Appraisals. See http://www.accurate-appraisal.com/faq.htm.
} 
is, some agents choose to purchase goods that they do not prefer because they anticipate that they will have to sell it later. Conformity is common - rather than being a knife-edge phenomenon, conformity is the typical outcome in the model. In the model the decision of whether or not to conform depends on the number of people with extreme preferences rather than the average preference. Because resale concerns rise with durability and the incidence of trade, there is greater conformity in markets for long-lived durables and in markets with frequent trade.

The equilibrium level of conformity in the model is usually not optimal. Surprisingly, the model suggests that there is typically too little conformity in durable goods markets. There are two reasons for this inefficiency. First, by conforming, agents reduce search costs. If they have a house that few others want, they will have difficulty selling it if they need to move. Of course the original builder has an incentive to conform to reduce the severity of these search costs. However, the search costs affect both buyers and sellers. Because the original owner only internalizes his own search costs, he has too little incentive to conform. Second, even when the house is sold, there is a possibility that the house will not be an ideal match for the new owner. This is a matching cost. The seller typically will not internalize the social costs incurred when someone "settles" for a house that is not ideal for their needs. Since it is more likely that he will be matched with someone who has a common preference, by conforming, the original owner could reduce the costs of such a mis-match. Only if the seller completely captures all of the surplus from the trade would he fully internalize this cost. Again, there is typically too little incentive to conform.

Finally, we consider the possibility of rent in the market for durable goods. By eliminating resale concerns, rent can potentially reduce conformity. The opposite can also happen. Agents who prefer uncommon types may choose to rent while people with typical preferences (the majority) choose to remain in the owneroccupied housing market. In this case, there is even greater incentive to conform in the owner-occupied market.

The classic example of conformity is perhaps the Keynesian beauty contest. Keynes originally compared financial investors with participants in a newspaper beauty contest. In most models of the Keynesian beauty contest, payoffs are assumed to depend on both one's own action and the average action of the other players. Models of this sort generate conformity because agents with intermediate preferences conform to the majority. Our model endogenizes the Keynesian beauty contest. While agents in our model do not care directly about the preferences or actions of other agents, in equilibrium they act as though they do. Because they 
buy and sell a common set of goods, durability and trade endogenously align the preferences of the agents.

The rest of the paper is organized as follows: Section 2 presents the model. In this section we describe optimal behavior and define and characterize equilibrium. Section 3 presents the main results of the paper. In this section we show that conformity increases with durability and the incidence of trade. Section 3 also considers welfare and the possibility of rent. Section 4 discusses the results and relates our paper to the existing literatures on durables, conformity, and optimal product diversity. Section 5 concludes.

\section{Model}

We consider a continuous-time matching model in which agents can own one of two types of a durable good. Although our analysis holds for any durable good, we assume that the good in our model is a house. We denote the two types of houses as type $a$ and $b$. Since we want to focus on heterogeneity in tastes, we assume that the costs of producing each type are equal. The houses could differ along many dimensions. For example, type $a$ houses could be "traditional-style" houses while type $b$ could be "modern-style" houses. Alternatively, type $a$ could be a two-story house with a large yard while type $b$ might be a one-story house with a small yard. Every agent must have a house in every period.

We normalize the utility functions so that all consumers get a flow utility of 1 from living in the type $a$ house. Consumers have different tastes for the type $b$ house. Specifically, each consumer has an individual taste parameter $z$ which quantifies their preference for type $b$ houses. For a consumer with taste parameter $z$ the flow utility from living in a type $b$ house is $1+z$. The flow utility for an agent with a given $z$ and a given house $x \in\{a, b\}$ is thus

$$
u_{z}(x)=\left\{\begin{array}{cl}
1 & \text { if } x=a \\
1+z & \text { if } x=b
\end{array} .\right.
$$

We assume that $z$ is distributed over the population according to a distribution function $F$.

From time to time agents switch houses. Agents may switch their house for one of two reasons. First, the house may "die". We think of the "death" of a house as capturing mainly normal depreciation but it may also include extreme idiosyncratic events such as fires, severe water damage, and so forth. When this occurs, the agent must build a new house. We refer to this event as the "build 
shock." An agent who gets the build shock decides which type of house to build and incurs a building cost $c$. The building cost is the same for all agents and for either type of house.

Second, the agent may be required to trade his house which we refer to as the "trade shock". Agents who get the trade shock must move out of their house and into a new house. To better motivate the trade shock, we imagine that each agent lives and works in one of two cities of equal size. Agents who get the trade shock have to move from one city to the other. When this happens the agent first has an opportunity to trade his house. The agent is matched randomly with a trading partner who is moving in the opposite direction. If both agents agree to trade, they simply exchange houses, otherwise the trade is rejected. If the trade fails, the agents are forced to scrap their old houses and build new houses of their choice. Let $\pi$ be the difference between the build cost $c$ and the scrap value of their old house. It is important to emphasize that agents are not trading because their preferences over houses change. They trade simply because they have to move from one city to the other. Thus, in the trade state, some agents will exchange houses of the same type (e.g., an $a$ for an $a$ ) as well as houses of different types.

Because $\pi$ is only incurred by agents who fail to trade, we refer to $\pi$ as the "trade penalty." One can alternatively interpret the trade penalty as reflecting other costs of buying and selling a house. For instance, one could think of $\pi$ as the expected cost of engaging in a protracted search in an environment with the possibility of re-matching. ${ }^{2}$ Under this interpretation $\pi$ would include the cost of renting while traders search for new houses and would also include the forgone interest on the sale price while a house waits to be sold. Sales commissions, fees and the costs of renovations could also be included in the trade penalty $\pi$.

If agents do not get the build or the trade shock they simply continue residing in their current house. We assume that the build shocks and trade shocks are independent Poisson processes with exogenous arrival rates $\delta$ and $\gamma$ respectively. Agents seek to maximize the discounted sum of flow utilities less costs. The discount rate is $r>0$. The next section analyzes the optimal behavior in this model.

\footnotetext{
${ }^{2}$ Allowing for agents to re-match greatly complicates the analysis. By ruling out this possibility, we gain tractability because the agent's trade decisions do not depend on the type of house they possess.
} 


\subsection{Optimal Policies and Conformity}

In this section we analyze the consumers maximization problem and present our definition of conformity. Throughout, we confine our attention to stationary equilibria. In a stationary equilibrium, consumers decide which types of houses to build and also which types of houses to accept in trade, taking the strategies of the other agents and the distribution of houses, both of which are time-invariant, as given. The main result in this section (Proposition 1) shows the form that the optimal policy takes. As we will demonstrate, the optimal policy is described by three critical values $z_{1}, z_{2}$, and $z_{3}$. These critical values define four intervals. In the first interval (agents with $z<z_{1}$ ), agents trade $a$ 's exclusively in the trading stage and build $a$ 's in the building stage. In the second interval $\left(z_{1}<z<z_{2}\right)$ agents build $a$ 's but accept either $a$ or $b$ in trade. In the third interval $\left(z_{2}<z<z_{3}\right)$ agents build $b$ 's and accept both $a$ or $b$ in trades. In the fourth interval $\left(z_{3}<z\right)$ agents trade $b$ 's exclusively and build $b$ 's. After analyzing the optimal trading and building decisions, in the next section, we turn to equilibrium.

We use standard dynamic programming techniques to analyze the agents' optimization problems. A policy (or strategy) for any agent consists of a decision rule in the trade state and a decision rule in the building state. The trade rule will specify whether an agent accepts or rejects a trade once he enters the trade state given the type of house he has. The build rule will specify which type of house the agent builds when he enters the build state.

Let $V(x ; z)$ be the value of following an optimal policy for a given agent with taste parameter $z$ who currently owns a house of type $x \in\{a, b\}$. Let $B(z)$ be the continuation value of entering the build state and let $T(x ; z)$ be the continuation value of entering the trading state when the agent has a type $x$ house. Because we are focusing on the behavior of a single agent, we suppress the argument $z$ in the following discussion. The value function satisfies ${ }^{3}$

$$
r V(x)=u(x)+\delta[B-V(x)]+\gamma[T(x)-V(x)] .
$$

\footnotetext{
${ }^{3}$ This value function can be obtained as the limit of a discrete-time dynamic programming environment. If $\Delta t$ is a discrete time interval, we could write the Bellman equation as

$$
\begin{aligned}
V(x)= & u(x) \Delta t+\frac{1}{1+r(\Delta t)} \times\{(1-\delta(\Delta t))(1-\gamma(\Delta t)) V(x) \\
& +(\delta(\Delta t)(1-\gamma(\Delta t))+\delta(\Delta t) \gamma(\Delta t))[B-V(x)]+\gamma(\Delta t)(1-\delta(\Delta t))[T(x)-V(x)]\}
\end{aligned}
$$

where we have assumed that if the agent simultaneously gets the build and trade shocks, he must build. Multiplying by $(1+r(\Delta t))$ and taking limits as $\Delta t \rightarrow 0$ gives equation (1).
} 
The continuation value of receiving the build shock is simply

$$
B=\max \{V(a), V(b)\}-c .
$$

The payoff to entering the trade stage $T(x)$ is more involved. In the trade stage, agents observe each other's houses but not their taste parameters. They then simultaneously choose to either accept or reject the trade. If they both agree to trade, they swap houses. If either disagrees, the trade is rejected. In this case, both traders pay the trade penalty $\pi$ and get new houses of their choice. (Recall that when a trade is rejected, the traders scrap their current houses and build new ones; $\pi$ is the build cost less the scrap value).

To compute $T(x)$, consider an agent who receives the trade shock and currently possesses a type $x$ house. Suppose that he is matched with someone with a type $y$ house. If the trade occurs, the agent gets the type $y$ house. His payoff in this case is simply $V(y)$. If either one rejects the trade then he selects a new house of his choice and pays the trade penalty. His payoff in this case is $\max \{V(a), V(b)\}-\pi$. Notice that the agent's trade decision is only relevant if his trading partner chooses to accept the trade. We therefore assume that agents accept trades whenever $V(y)>\max \{V(a), V(b)\}-\pi$ and reject otherwise. ${ }^{4}$ Notice also that neither $V(y)$ nor $\max \{V(a), V(b)\}-\pi$ depend on $x$. Thus, trade decisions are independent of the type of house the agent possesses when he enters the trade stage.

It is easy to show that if an agent builds type $x \in\{a, b\}$ then he also accepts $x$ in trade. To see this, first note that because the agent chooses to build $x$, it must be the case that $V(x)=\max \{V(a), V(b)\}$. As a result, he also chooses $x$ whenever he gets the trade shock and the trade is rejected. Thus, if he is offered $x$ in trade, he gets $V(x)$ if he accepts the offer and $V(x)-\pi$ if he declines the offer. For future reference, we present this observation as a Lemma. All proofs are in the appendix.

Lemma 1. If an agent builds type $x \in\{a, b\}$, then he also accepts type $x$ in trade.

An immediate implication of Lemma 1 is that no agent rejects all houses in trade. In addition, because an agent's trading decisions are independent of his trading partner's taste parameter and also independent of the house he owns, there

\footnotetext{
${ }^{4}$ This assumption rules out the trivial and uninteresting equilibrium in which agents always reject every trade.
} 
are only three relevant trading rules to consider: (1) accept $a$ only; (2) accept $b$ only; or (3) accept either $a$ or $b$. We denote these trading rules simply as $a, b$, and $a b$. We refer to agents who accept only a particular type of house (either $a$ or $b$ but not both) as exclusive traders. Agents who play $a b$ and thus accept both types are said to be inclusive traders.

Our aim is to express the value functions $V(x), T(x)$ and $B$ in terms of the policies of the other agents. To do this, we first define $\lambda(y, \tau)$ as the probability of being matched with someone who has a type $y$ house and who follows trading rule $\tau \in\{a, b, a b\} .^{5}$ For example, $\lambda(a, a b)$ is the probability of meeting someone who possesses a type $a$ house and follows trading rule $a b$. Agents who accept type $x \in\{a, b\}$ houses in trade either follow the exclusive trading rule $x$ or the inclusive trading rule $a b$. The probability of meeting an agent who accepts a type $x$ house in trade is therefore $\sum_{\tau \in\{x, a b\}} \sum_{y \in\{a, b\}} \lambda(y, \tau)$. We can now write the expected value of entering the trading state with a type $x$ house as

$$
\begin{aligned}
T(x)= & \sum_{\tau \in\{x, a b\}} \sum_{y \in\{a, b\}}(\lambda(y, \tau) \max \{V(y), \max \{V(a), V(b)\}-\pi\}) \\
& +\left(1-\sum_{\tau \in\{x, a b\}} \sum_{y \in\{a, b\}} \lambda(y, \tau)\right)(\max \{V(a), V(b)\}-\pi)
\end{aligned}
$$

Because we can express the continuation values $T(x)$ and $B$ in terms of the value function $V(x)$ and the matching probabilities $\lambda(y, \tau)$, equation (1) implicitly defines the value function itself solely in terms of the underlying matching probabilities. Given any set of values $V(a)$ and $V(b)$, and fixed trading probabilities, equation (3) implies associated values $T(a)$ and $T(b)$ and equation (2) implies an associated value $B$. Equation (1) then implies a new set of values $\hat{V}(a), \hat{V}(b)$. It is straightforward to show that this mapping satisfies Blackwell's sufficient conditions for a contraction mapping and thus has a unique fixed point. We summarize this in the following Lemma:

Lemma 2. Given matching probabilities $\lambda(x, \tau)$ for $x \in\{a, b\}$ and $\tau \in\{a, b, a b\}$ with $\sum_{\tau \in\{a, b, a b\}} \sum_{y \in\{a, b\}} \lambda(y, \tau)=1$ there exist unique values $V(a), V(b), T(a)$, $T(b)$, and $B$ satisfying (1), (2), and (3).

\footnotetext{
${ }^{5}$ In a stationary equilibrium, $\lambda(x, \tau)$ will be uniquely determined by the strategies of the other agents.
} 
An optimal policy in our model consists of a building rule (whether to build $a$ or $b$ ) and a trading rule (whether to accept $a, b$ or both). Because the continuation values in Lemma 2 are fixed numbers, the optimal policy is stationary.

We now characterize the optimal policy. We first note that there are only four relevant policies. To see this note that if you follow the trade rule $a$ (an exclusive trading rule) then by Lemma 1 you must build a type $a$ house in the build stage. Similarly, if you are an exclusive $b$ trader, you build type $b$. Thus, without loss of generality we can confine our attention to four possible policies: build $a$ and follow trade rule $a$; build $a$ and follow trade rule $a b$; build $b$ and follow trade rule $b$ or build $b$ and follow trade rule $a b$. We can write these policies compactly as $(x, \tau)$ where $x \in\{a, b\}$ and $\tau \in\{a, b, a b\}$.

Agents with different taste parameters $z$ choose different policies. Intuitively, agents with sufficiently negative $z$ 's (who strongly dislike type $b$ houses) build type $a$ houses and accept only type $a$ houses in trade. Similarly, agents with sufficiently positive $z$ 's (who have strong preferences for $b$ houses) build type $b$ and accept only type $b$ in trade. Agents with intermediate values of $z$ do not have strong preferences for either type and thus accept either $a$ or $b$ in trade. The following proposition formalizes this intuition. Specifically, it shows that the optimal policy for any agent is determined by three cutoff values $z_{1}, z_{2}$, and $z_{3}$. These cutoffs define four intervals. In the first interval agents play $(a, a)$ (they accept only $a$ in trade and build $a$ when they get the build shock). In the second interval agents play $(a, a b)$. In the third interval agents play $(b, a b)$, and in the last interval agents play $(b, b)$.

Proposition 1. Given non-negative $\lambda(a, a), \lambda(b, b), \lambda(a, a b)$, and $\lambda(b, a b)$ summing to 1 , define $z_{1}, z_{2}$, and $z_{3}$ as follows:

$$
\begin{gathered}
z_{1}=-\pi\{r+\delta+\gamma[1-\lambda(a, a)]\} \\
z_{2}=\gamma \pi[\lambda(a, a)-\lambda(b, b)] \\
z_{3}=\pi\{r+\delta+\gamma[1-\lambda(b, b)]\}
\end{gathered}
$$

Then, for any $z<z_{1}$ the optimal strategy is $(a, a)$; for $z_{1}<z<z_{2}$ the optimal strategy is $(a, a b)$; for any $z_{2}<z<z_{3}$ the optimal strategy is $(b, a b)$ and for $z>z_{3}$ the optimal strategy is $(b, b)$.

Notice that the cutoffs depend only on the number of exclusive traders of each type. The precise distribution of tastes of other traders is not relevant once $\lambda(a, a)$ 
and $\lambda(b, b)$ are given. In particular, the average taste parameter is unimportant. The building decisions of the other agents is also irrelevant. Whether the inclusive traders build more $a$ 's or more $b$ 's has no bearing on an individual's decision. It is important to understand the economic intuition behind this proposition. Because the logic is identical for the $b$ cutoff $\left(z_{3}\right)$ we focus on the determination of the $a$ cutoff $\left(z_{1}\right)$.

Consider an agent with a taste parameter $z$ less than zero who optimally follows an $(a, a)$ policy. Suppose that he receives the trade shock and is matched with someone who has a $b$ and considers a "one-shot" deviation from his optimal policy. Specifically, suppose that he decides to accept the $b$ house but then revert to the trade rule $a$ for subsequent trade shocks. There are costs and benefits to this deviation. The benefit is that he will avoid the immediate trade penalty $\pi$. There are two costs. First, he will reside in a house other than his preferred type for some time. This expected loss is $z /(r+\delta+\gamma)$ (recall that $z<0)$. Second, he may encounter someone who follows an exclusive $a$ trading rule while he still has the $b$ house. In this case he pays the trade penalty $\pi{ }^{6}$ The expected cost is $\pi \gamma \lambda(a, a) /(r+\delta+\gamma)$. The discount rate $(r+\delta+\gamma)$ reflects both the agents impatience and the likelihood of moving out of the type $b$ house (which occurs if the house "dies" or if he gets another trade shock). There are no additional costs because if the agent is matched with any other type of trader, then the trade penalties are the same as if he continued to follow the $(a, a)$ policy. With probability $\lambda(b, a b)+\lambda(b, b)$ he declines the trade (which he would do under the $(a, a)$ policy) and with probability $\lambda(a, a b)$ he accepts the trade (which he would do under the $(a, a)$ policy). Thus, we can write the net benefit of this deviation as

$$
\pi+\frac{z}{r+\delta+\gamma}-\pi \frac{\gamma \lambda(a, a)}{r+\delta+\gamma} \leq 0
$$

The inequality follows because we assumed that it was optimal to follow $(a, a)$. If the agent is indifferent between $(a, a)$ and $(a, a b)$, this expression would hold with equality. Rearranging this expression shows that the expression is zero only if $z=z_{1}$ as given in the Proposition. ${ }^{7}$

Note that the final component of (4) depends on $\lambda(a, a)$. If $\lambda(a, a)$ is high then many people turn down $b$ 's in the trade stage. The higher is $\lambda(a, a)$, the

\footnotetext{
${ }^{6}$ Note that he would not experience this trade penalty if he did not deviate from the $(a, a)$ strategy.

${ }^{7}$ Note that the agent could alternatively consider a deviation in which he accepts $b$ and continues to follow the policy $(a, a b)$ until he again finds himself holding a type $a$ house. This deviation is somewhat more complicated but results in the same cutoff. The costs and benefits
} 
more costly it is to follow the $(a, a b)$ policy. There is an important feedback effect to this logic. If I believe that many people decline $b$ 's in trade then I will decline $b$ 's. If enough people behave as I do, this will confirm my beliefs. This "positive feedback" effect introduces the possibility of multiple equilibria - a possibility we will demonstrate in the next section.

Given $\lambda(a, a), z_{1}$ is closer to zero the closer $r, \delta$ and $\gamma$ are to 0 . Thus, given $z$, an agent is more likely to be exclusive if the object is very durable (low $\delta$ ), if the agent is very patient (low $r$ ), and if it is unlikely that he will trade (low $\gamma$ ). Intuitively, if the object is very durable and you are not likely to trade it, and you care a lot about the future, then you don't want to get stuck with the wrong house.

Like the trading cutoffs $z_{1}$ and $z_{3}$ the building cutoff $z_{2}$ is also determined by the number of extreme traders. In particular, $z_{2}$ is determined by the difference $\lambda(a, a)-\lambda(b, b)$. If $\lambda(a, a)>\lambda(b, b)$ then $z_{2}>0$. Note that $z_{1}+z_{3}=z_{2}$. Only if $\lambda(a, a)$ and $\lambda(b, b)$ are exactly equal will $z_{2}=0$. The $z_{2}$ cutoff is of special interest because it determines the equilibrium number of houses of each type built. If $z_{2}>0$ for instance, there may be people who prefer $b$ houses but build $a$ 's when they get the build shock (i.e., for whom $0<z<z_{2}$ ). We say that if an agent builds a house other than the type dictated by his taste parameter $z$, then the agent is conforming to the market.

Definition 1. If $z_{2} \neq 0$ then we say that there is conformity in the market. If $z_{2}>0$ then the market conforms on type a houses, and if $z_{2}<0$ then the market conforms on type $b$ houses. If $z_{2}=0$ then we say that there is no conformity.

To summarize, the optimal policy - what types should an agent accept in trade and what type should the agent build - depends on the behavior of agents with extreme preferences for each types of house rather than the behavior of the typical agent. Specifically, whether or not an agent conforms depends on the

are

$$
\begin{gathered}
\underbrace{\frac{-z}{r+\delta+\gamma[\lambda(a, a b)+\lambda(a, a)]}+\pi \frac{\gamma \lambda(a, a)}{r+\delta+\gamma[\lambda(a, a b)+\lambda(a, a)]}}_{\text {Costs }} \\
\underbrace{\pi+\pi \frac{\gamma(\lambda(b, a b)+\lambda(b, b))}{r+\delta+\gamma[\lambda(a, a b)+\lambda(a, a)]}}_{\text {Benefits }}
\end{gathered}
$$

Equating these terms reveals that the critical $z$ is again $z_{1}$ as in Proposition 1 . 
relative number of exclusive traders of $a$ and $b$ houses. If there are many people who accept only $a$ houses then there is a strong incentive to reject $b$ houses in trade and to build $a$ houses in the build stage even if you enjoy living in the $b$ house more than the $a$ house. The dependence on other traders is greater if the durable is very long-lived, if trade is likely, and if the agents are very patient.

We now turn to the solution of the model in which we define and characterize the equilibrium.

\subsection{Equilibrium}

Before we provide a definition of an equilibrium, we first describe the relationship between the policies followed by the agents and the matching probabilities implied by the policies. Proposition 1 implies that optimal policies are chosen according to three cutoff values $z_{1}, z_{2}$, and $z_{3}$. The number of people who follow policy $(a, a)$ is $F\left(z_{1}\right)$; the number who follow $(a, a b)$ is $F\left(z_{2}\right)-F\left(z_{1}\right)$; the number who follow $(b, a b)$ is $F\left(z_{3}\right)-F\left(z_{2}\right)$ and the number who follow $(b, b)$ is $1-F\left(z_{3}\right)$. We will use this information to compute the implied matching probabilities below. This computation is more delicate than it might seem. While it is true that in equilibrium, the number of people who follow policy $(a, a)$ or policy $(b, b)$ is the same as $\lambda(a, a)$ and $\lambda(b, b)$, the difficulty arises because typically the number of people who follow policy $(a, a b)$ or policy $(b, a b)$ is different than $\lambda(a, a b)$ and $\lambda(b, a b)$. (Thus for example $F\left(z_{2}\right)-F\left(z_{1}\right)$ is different than $\lambda(a, a b)$.) The reason that these numbers differ is that often one type of house will be rejected more often than the other type. This effectively causes a difference between the distribution of houses for sale and the distribution of houses built. In fact, there are three potentially distinct distributions of houses in our model: the distribution of houses built, the distribution of houses in existence and the distribution of houses for sale on the market. Because the houses for sale are a random selection from the existing housing stock and because houses do not remain on the market after their initial match, the distribution of houses on the market is the same as the distribution of houses in existence. When a trade is rejected however, agents effectively transform their current house into the type of house they want (at the cost $\pi$ ). If one house is rejected more frequently than the other, then the distribution of houses in existence will differ systematically from the distribution of houses built. For instance, if type $b$ houses are rejected more frequently than $a$ houses, the steady state number of $b$ houses in existence (and for sale on the market) will be less than the number of $b$ houses built. 
In fact, the distribution of houses built often differs from the distribution of houses for sale in durable goods models with matching frictions. For instance, if we assumed that houses remained on the market until they were sold, then if $b$ houses were rejected more often than $a$ houses, the housing market would have relatively more $b$ houses waiting to be sold. In this case, there would again be a difference between the distribution of houses built and houses for sale. Unlike our model however, in this case the distribution of existing houses would be the same as the distribution of houses built because once a house is built, it would remain in use until it depreciated. Indeed, there is a natural analog to this phenomenon in the real world. Because houses that are difficult to sell remain vacant for some time, the distribution of houses built is not the same as the distribution of houses for sale. ${ }^{8}$

Now we turn to the computation of the matching probabilities for any given set of cutoffs $z_{1}, z_{2}$, and $z_{3}$. Denote the number of people who follow policy $s$ and hold type $x$ as $P_{s}(x)$. Thus $x$ is either $a$ or $b$ while a policy $s \in$ $\{(a, a),(b, b),(a, a b),(b, a b)\}$. Agents who follow exclusive trading rules always hold the same type of house that they build. As a result $P_{a, a}(b)=P_{b, b}(a)=0$ and

$$
\begin{gathered}
P_{a, a}(a)=\lambda(a, a)=F\left(z_{1}\right) \\
P_{b, b}(b)=\lambda(b, b)=1-F\left(z_{3}\right)
\end{gathered}
$$

Traders who follow inclusive trading rules sometimes live in houses other than the type they build. To illustrate, consider agents who play strategy $(a, a b)$. $P_{a, a b}(a)$ of these agents hold $a$, and $P_{a, a b}(b)$ of them hold $b$. Moreover, $P_{a, a b}(a)+$ $P_{a, a b}(b)$ equals the total number of people playing strategy $(a, a b)$, i.e., $F\left(z_{2}\right)-$ $F\left(z_{1}\right)$. Because people who play $(a, a b)$ always acquire $a$ houses when they get the build shock or when a trade is declined, the only way for them to acquire a $b$ is to get a trade shock and match with someone who has $b$ and accepts $a$. By Lemma 1 these people must be playing an inclusive (i.e. $a b$ ) trading strategy. There are $P_{a, a b}(b)+P_{b, a b}(b)$ such traders. Thus, the flow out of $P_{a, a b}(a)$ is

$$
\gamma P_{a, a b}(a)\left(P_{a, a b}(b)+P_{b, a b}(b)\right) .
$$

The flow into $P_{a, a b}(a)$ consists of people in $P_{a, a b}(b)$ who either (1) get a trade shock and match with someone who has $a$ and plays an inclusive trading strategy, or (2) get a trade shock and match with someone who plays strategy $(a, a)$ or $(3)$

\footnotetext{
${ }^{8}$ Recall that one interpretation of the trade penalty in our model is a cost due to vacancy.
} 
get the build shock (and thus build $a$ ). Thus, the flow into $P_{a, a b}(a)$ is

$$
P_{a, a b}(b)\left[\gamma\left(P_{a, a b}(a)+P_{b, a b}(a)+P_{a, a}(a)\right)+\delta\right]
$$

In steady state the inflows and outflows exactly offset. This requires

$$
\gamma P_{a, a b}(a) P_{b, a b}(b)=P_{a, a b}(b)\left[\gamma\left(P_{b, a b}(a)+P_{a, a}(a)\right)+\delta\right]
$$

Similar relationships hold for people who play $(b, a b)$. These equations can be solved for the steady state values of $P_{a, a b}(a), P_{a, a b}(b), P_{b, a b}(a)$, and $P_{b, a b}(b)$ as functions of $z_{1}, z_{2}$ and $z_{3}$. These values give us the implied matching probabilities. We summarize these relationships in the following Lemma.

Lemma 3. Let $z_{1}, z_{2}$ and $z_{3}$ be given. Define

$$
r_{a}=\frac{\delta+\gamma F\left(z_{1}\right)}{F\left(z_{3}\right)-F\left(z_{2}\right)}, r_{b}=\frac{\delta+\gamma\left[1-F\left(z_{3}\right)\right]}{F\left(z_{2}\right)-F\left(z_{1}\right)}
$$

then the steady state values of $P_{a, a b}(a), P_{a, a b}(b), P_{b, a b}(a)$, and $P_{b, a b}(b)$ are given by

$$
\begin{aligned}
P_{a, a b}(b) & =\frac{\gamma r_{b}}{\gamma r_{a}+\gamma r_{b}+r_{a} r_{b}}\left(F\left(z_{2}\right)-F\left(z_{1}\right)\right) \\
P_{a, a b}(a) & =\frac{\gamma r_{a}+r_{a} r_{b}}{\gamma r_{a}+\gamma r_{b}+r_{a} r_{b}}\left(F\left(z_{2}\right)-F\left(z_{1}\right)\right) \\
P_{b, a b}(a) & =\frac{\gamma r_{a}}{\gamma r_{a}+\gamma r_{b}+r_{a} r_{b}}\left(F\left(z_{3}\right)-F\left(z_{2}\right)\right) \\
P_{b, a b}(b) & =\frac{\gamma r_{b}+r_{a} r_{b}}{\gamma r_{a}+\gamma r_{b}+r_{a} r_{b}}\left(F\left(z_{3}\right)-F\left(z_{2}\right)\right)
\end{aligned}
$$

and the steady state matching probabilities are

$$
\begin{gathered}
\lambda(a, a)=F\left(z_{1}\right) \\
\lambda(b, b)=1-F\left(z_{3}\right) \\
\lambda(a, a b)=P_{a, a b}(a)+P_{b, a b}(a) \\
\lambda(b, a b)=P_{a, a b}(b)+P_{b, a b}(b)
\end{gathered}
$$


An equilibrium is a fixed point in the matching probabilities. Given the perceived matching probabilities, agents follow optimal policies. The matching probabilities are, in turn, implied by the policies chosen as described in Lemma 3. This leads to a mapping from perceived probabilities to implied probabilities. An equilibrium is a fixed point of this mapping. We now present a formal definition of a steady state equilibrium.

Definition 2. A steady state equilibrium consists of four non-negative numbers $\lambda(a, a), \lambda(b, b), \lambda(a, a b)$, and $\lambda(b, a b)$ summing to one, and three cutoffs $z_{1}, z_{2}$ and $z_{3}$ such that

1. Given $\lambda(a, a), \lambda(b, b), \lambda(a, a b)$, and $\lambda(b, a b)$, Proposition 1 implies the cutoffs $z_{1}, z_{2}$ and $z_{3}$.

2. Given $z_{1}, z_{2}$ and $z_{3}$, Lemma 3 implies $\lambda(a, a), \lambda(b, b), \lambda(a, a b)$, and $\lambda(b, a b)$.

To prove that an equilibrium exists, note that the cutoffs for the exclusive $a$ and $b$ traders $\left(z_{1}\right.$ and $\left.z_{3}\right)$ are each governed only by the number of exclusive $a$ and $b$ traders respectively. More precisely, $z_{1}$ is completely defined once $\lambda(a, a)=F\left(z_{1}\right)$ is given. Similarly, $z_{3}$ depends on only $\lambda(b, b)=1-F\left(z_{3}\right)$. This implies that we can analyze the determination of these cutoffs separately. Define two mappings $L_{a, a}:[0,1] \rightarrow[0,1]$ and $L_{b, b}:[0,1] \rightarrow[0,1]$ as follows: Let $\lambda(a, a) \in[0,1]$ and $\lambda(b, b) \in[0,1]$ be given. Set

$$
L_{a, a}(\lambda(a, a))=F(-\pi\{r+\delta+\gamma[1-\lambda(a, a)]\}) \in[0,1]
$$

and

$$
L_{b, b}(\lambda(b, b))=1-F(\pi\{r+\delta+\gamma[1-\lambda(b, b)]\}) \in[0,1] .
$$

While these mappings may not be continuous (which would occur if $F$ had mass points at certain $z$ 's for instance), they are both increasing functions on a compact set which implies that each has at least one fixed point.

Lemma 4. The mappings $L_{a, a}$ and $L_{b, b}$ defined by (5) and (6) each have at least one fixed point.

Any combination of fixed points of these mappings correspond to equilibrium values of $\lambda(a, a)$ and $\lambda(b, b)$. To complete the construction of an equilibrium, given any fixed points $\lambda(a, a)$ and $\lambda(b, b)$, define $z_{1}, z_{2}$ and $z_{3}$ as in Proposition 
1 and calculate $\lambda(a, a b)$ and $\lambda(b, a b)$ as in Lemma 3. The resulting matching probabilities $\lambda(a, a), \lambda(b, b), \lambda(a, a b)$, and $\lambda(b, a b)$, and cutoffs $z_{1}, z_{2}$ and $z_{3}$ satisfy the definition of a steady state equilibrium. This establishes the following proposition:

Proposition 2. Given any distribution of types $F$, there exists at least one steady state equilibrium.

Since each mapping may have more than one fixed point, there may be multiple steady state equilibria. We now consider two examples. Example 1 illustrates a case in which there is a unique equilibrium and the market conforms to the average taste. Example 2 shows that conformity may arise due to the multiplicity of equilibria even when the distribution is symmetric around zero, so that the average consumer is indifferent between the two types.

Example 1 Suppose that $F$ is uniform on the interval $[-q+\mu, q+\mu]$. We assume that the distribution is wide in the sense that $-q+\mu<-\pi(r+\delta+\gamma)$. Figure 1 shows the fixed points mappings $L_{a, a}$ and $L_{b, b}$ for different values of the mean taste $\mu$. The light dashed line corresponds to $\mu=0$. In this case, the distribution is symmetric around zero and thus $L_{a, a}=L_{b, b}$. In the unique equilibrium $\lambda(a, a)=\lambda(b, b)$ and $z_{2}=0$. Thus there is no conformity in equilibrium. The dark lines correspond to the case in which $\mu>0$. Because $F$ has shifted to the right, $L_{b, b}$ has shifted up while $L_{a, a}$ has shifted down relative to the light dashed line. In the resulting equilibrium $\lambda(a, a)<\lambda(b, b)$ and $z_{2}<0$ so that the market conforms on type $b$.

Example 2 Suppose that $F$ is symmetric about 0 but not uniform. The mappings $L_{a, a}$ and $L_{b, b}$ are identical (as in the first example when $\mu=0$ ). Figure 2 shows an example in which this mapping has multiple fixed points. Note that by symmetry $F(0)=1 / 2$ and thus the mapping is bounded above by $1 / 2$. As it is drawn there are three fixed points $\lambda_{1}^{*}<\lambda_{2}^{*}<\lambda_{3}^{*}$. By setting $\lambda(a, a)=\lambda_{i}^{*}$ and $\lambda(b, b)=\lambda_{j}^{*}(i, j=1$ or 2$)$ we can construct nine possible equilibria. Three of these are non-conforming equilibria (when $\lambda(a, a)=\lambda(b, b))$ and the other six are conforming equilibria (when $\lambda(a, a) \neq \lambda(b, b))$.

Clearly conformity is a generic property of equilibrium in our model. Nonconforming equilibria only occur in knife-edge cases in which $\lambda(a, a)=\lambda(b, b)$ 
while conforming equilibria occur in all other cases. In the next section we show that markets for highly durable goods in which trade is common have a strong tendency to conform.

\section{Results and extensions}

In this section we analyze the relationship between conformity and the underlying parameters of the model. We pay particular attention to durability and the frequency of trade. We then discuss the welfare properties of the equilibrium. Finally, we consider the implications of allowing for a rental market that coexists with the owner-occupied market.

\subsection{Comparative Statics}

In this section we consider how variations in the durability of the good, the frequency of the trade shocks, the subjective time discount factor and the trade penalty affect the equilibrium. To facilitate the analysis, we place restrictions on the distribution $F$ to rule out multiple equilibria. The following assumption provides sufficient conditions for a unique equilibrium.

Assumption $1 F$ has a density function $f$ which is symmetric about the mean $\mu$, quasi-concave and single peaked with $f(\mu)<\frac{1}{\pi \gamma}$.

The following proposition states the main result in this section.

Proposition 3. If $F$ satisfies Assumption 1 then

1. The equilibrium is unique.

2. The market conforms to the mean taste whenever $\mu \neq 0$ (i.e., the market conforms on $a$ if $\mu<0$ and conforms on $b$ if $\mu>0$ ).

3. If $\mu \neq 0$, an increase in durability (lower $\delta$ ), patience (lower $r$ ), the likelihood of trade (higher $\gamma$ ) or the trade penalty (higher $\pi$ ) causes conformity to increase.

Part 1 of the proposition (uniqueness) follows from the bound on $f$ in Assumption 1 which ensures that the fixed point mappings $L_{a, a}$ and $L_{b, b}$ never have slopes greater than 1 . Part 2 of Proposition 3 demonstrates that conformity is a 
generic property within this class of distributions and is therefore to be expected in equilibrium. While the market conforms to the average taste, it does so because there are more exclusive traders on that side of the market. The third result in the proposition demonstrates that conformity increases with patience, the trade penalty, durability, and the incidence of trade.

If $\delta=\infty$ then the good is a non-durable. In this case, there is no conformity in equilibrium. Since the good does not survive beyond the immediate period in which it is produced and sold, there is no concern about its resale value. As a result, consumers purchases of nondurables should be a pure reflection of their own idiosyncratic valuations of the goods. For lower values of $\delta$, resale becomes more of a concern. Because the highest depreciation objects will be held by a wide variety of people, their features should better reflect the average valuation of the good rather than just the tastes of the initial purchaser.

If $\gamma=0$ then the good is never traded. In this case, again there is no incentive to conform. Once the good is purchased, an agent will retain it until the good falls apart. Therefore, when he decides which type to purchase, he should take into account only his own preferences. For higher values of $\gamma$, there is an increased incentive to conform to the average taste. This is due naturally to the increased likelihood of trade. While the intuition for this result is natural, it seems to stand in stark contrast to free-market folklore - rather than encouraging market diversity, increased trade in durable goods encourages market conformity.

High $\gamma$ and low $\delta$ affect conformity in the same way. Intuitively, if the durable is traded frequently then it is important to have a durable which is valued by the other traders. Holding the probability of trade fixed, increasing the durability of the good extends its expected service life. As a result, there are more opportunities for it to change hands. By the same token, holding the depreciation rate fixed, increasing the likelihood of trade also causes the good to be exchanged more often.

The intuition for the effects of $r$ and $\pi$ on conformity is also natural. Higher trade penalties increase the cost of having trades rejected and thus increase the

incentive to conform. If agents have low discount rates the future possibility of resale weighs more heavily on their current purchasing decisions which again increases the incentive to conform.

\subsection{Welfare}

The previous section shows that in the case of durable goods, conformity is the rule rather than the exception. In this section, we consider the welfare implications 
of conformity. While conformity has an obvious cost - people live in houses that they don't prefer - it also has benefits. Conformity lowers search costs by reducing the probability of having a trade rejected. Naturally, social welfare is lower when houses are vacant or when people are forced to conduct protracted searches for a good match. Furthermore, if houses are long-lived, the original owner will almost surely transfer the home to another agent who may or may not like that type of house. The potential mismatch of preferences and allocations is a cost to society that conformity mitigates by increasing the value of the home for subsequent home owners. As a result, we cannot conclude that conformity is inefficient. A social planner may want some degree of conformity.

Below, we explore the welfare implications of conformity in our model. We do this by considering examples that illustrate the costs and benefits of conformity. Surprisingly, the examples suggest that often there is too little conformity in equilibrium. The first two examples isolate the two benefits that come from conformity. In each case, the benefits to conformity outweigh the costs. We then present an example that demonstrates that there can be too much conformity. This happens when the two benefits work in opposite directions.

We assume that a social planner values the flow utility of all agents equally and seeks to maximize the steady state flow of utility in each period. The following Lemma provides an expression for flow of social welfare in the steady state.

Lemma 5. For any given cutoffs $z_{1}, z_{2}$ and $z_{3}$, let $P_{a, a b}(a), P_{b, a b}(a), P_{a, a b}(b)$ and $P_{b, a b}(b)$ be given as in Lemma 3 and define

$$
\psi_{a, a b}=\left(\frac{P_{a, a b}(a)}{F\left(z_{2}\right)-F\left(z_{1}\right)}\right) \text { and } \psi_{b, a b}=\left(\frac{P_{b, a b}(b)}{F\left(z_{3}\right)-F\left(z_{2}\right)}\right) .
$$

Then, the flow of social welfare is

$$
\begin{aligned}
W\left(z_{1}, z_{2}, z_{3}\right) & =\{1-\pi \gamma[\lambda(b, b)+\lambda(b, a b)]\} F\left(z_{1}\right) \\
& +\int_{z_{1}}^{z_{2}}\left\{\psi_{a, a b}[1-\pi \gamma \lambda(b, b)]+\left(1-\psi_{a, a b}\right)[1+z-\pi \gamma \lambda(a, a)]\right\} d F(z) \\
& +\int_{z_{2}}^{z_{3}}\left\{\left(1-\psi_{b, a b}\right)[1-\pi \gamma \lambda(b, b)]+\psi_{b, a b}[1+z-\pi \gamma \lambda(a, a)]\right\} d F(z) \\
& +\int_{z_{3}}^{\infty}\{1+z-\pi \gamma[\lambda(a, a)+\lambda(a, a b)]\} d F(z)-\delta c .
\end{aligned}
$$

Each term in the welfare function has a natural economic interpretation. The first term is the flow of welfare to the $(a, a)$ traders. Since they are exclusive 
traders, they always hold type $a$ houses and thus they all receive a flow utility of 1. In addition, they sometimes incur the trade penalty by matching with $(b, b)$ and $(b, a b)$ traders. The second term is the flow of welfare for agents who follow the $(a, a b)$ policy. These individuals sometimes hold $a$ houses but sometimes hold $b$ 's. The fraction of time these agents hold the $a$ house is $\psi_{a, a b}$. In this case, their flow utility is 1 less the trade hazard of meeting an exclusive $b$ trader. The remaining fraction of time $\left(1-\psi_{a, a b}\right)$ they hold $b$ houses. In this case, their flow utility is $1+z$ less the trade hazard of meeting an exclusive $a$ trader. The remaining terms have analogous interpretations. Notice that all agents are equally likely to experience the depreciation shock so all welfare flows are reduced by $\delta c$.

We now consider the welfare implications of increasing or decreasing conformity. Conformity can increase welfare through two separate channels. First, increased conformity can reduce the incidence of the trade penalty. Second, increased conformity means that the average type resides in their preferred house more often. The following two examples illustrate these effects.

One Inclusive and One Exclusive Type One of the costs associated with insufficient conformity is that trades will be declined too often. This happens because in equilibrium, agents internalize only their own trade penalties and not those of their trading partners. Agents at the $z_{2}$ cutoff are indifferent between building either type of house. For these agents, the benefits to living in their preferred houses if they choose not to conform, is exactly offset by the benefits of reducing the incidence of the trade penalty if they choose to conform. Because the house they build will be rejected by one group of exclusive traders, these agents impose a negative trade externality on one group of exclusive traders and a positive trade externality on the other. Since there are more exclusive traders on the conforming side of the market, social welfare would rise if the marginal agents conformed.

The following example isolates this effect. Suppose there are two types of agents. Call these types "medium", and "high". The high type has a strong preference for $b$ while the medium type has a weak preference for $a$. We write their $z$ 's as $z^{M}$ and $z^{H}$ respectively. We choose $z^{H}$ to ensure that the high traders play an exclusive trading strategy in equilibrium (i.e., they play $(b, b)) .{ }^{9}$ The medium agents may or may not conform. The number of medium agents is $\alpha$ and the number of high agents is $(1-\alpha)$. In the equilibrium, $\lambda(b, b)=(1-\alpha)$ and

\footnotetext{
${ }^{9}$ The biggest $z_{3}$ can be is $\pi(r+\delta)$. If $z^{H}>\pi(r+\delta)$, then the high types will play $(b, b)$ in any equilibrium.
} 
there is conformity on $b$. Specifically, the building cutoff is

$$
z_{2}=-\gamma \pi(1-\alpha)<0
$$

In the market equilibrium, the medium types decide to follow strategy $(a, a b)$ if $z^{M}<z_{2}$ and follow $(b, a b)$ otherwise. ${ }^{10}$ To determine whether increased conformity is socially desirable, we compute the social welfare when the medium types play strategy $(a, a b)$ and compare it to the welfare when they play $(b, a b)$.

If the medium agents follow strategy $(a, a b)$, then $F\left(z_{2}\right)-F\left(z_{1}\right)=\alpha$ and the medium types never trade with the high types. In this case, $P_{a, a b}(a)=\alpha$ and $P_{a, a b}(b)=P_{b, a b}(a)=P_{b, a b}(b)=0$. Using Lemma 5, welfare is

$$
W_{a, a b}=1+z^{H}(1-\alpha)-2 \pi \gamma \alpha(1-\alpha)-\delta c
$$

where the subscript $a, a b$ indicates that the medium types play strategy $(a, a b)$. On the other hand, if the medium agents follow strategy $(b, a b)$ then by similar calculations welfare is

$$
W_{b, a b}=1+\alpha z^{M}+(1-\alpha) z^{H}-\delta c .
$$

Strategy $(a, a b)$ is socially preferable to $(b, a b)$ if $W_{a, a b} \geq W_{b, a b}$. It is straightforward to show that this requires

$$
-2 \pi \gamma(1-\alpha) \geq z^{M}
$$

Recalling that in equilibrium $z_{2}=-\gamma \pi(1-\alpha)$ the social optimum requires

$$
2 z_{2} \geq z^{M}
$$

Put differently, the socially optimal policy calls for twice as much conformity as in the market equilibrium.

The reason for conforming in this case is to avoid the trade penalty. If the medium agents are close to the equilibrium $z_{2}$ then they are balancing the benefits of having the type of house they prefer with the costs of incurring the trade penalty more often. Because they do not care about the trade penalty for the other agents, they are too reluctant to conform to the majority type. The social planner internalizes both trade penalties every time a trade is declined and thus sets a cutoff that is twice the equilibrium cutoff.

\footnotetext{
${ }^{10}$ Note that because the high types follow an exclusive strategy, for the medium types, there is no operational distinction between strategies $(a, a)$ and $(a, a b)$ and between $(b, b)$ and $(b, a b)$.
} 
Two inclusive types The previous example showed that, because conforming to the market reduces trade penalties, and because agents only internalize their own trade penalties, there can be too little conformity in equilibrium. This example highlights another source of inefficiency caused by too little conformity. If agents are willing to trade (i.e., they are inclusive), then conforming implies that your trading partners will live in their preferred houses more often. Because agents only care about the houses they live in, conformity can again be inefficiently low in equilibrium.

Suppose there are two types "low" and "high". We assume that $-\pi(r+\delta)<$ $z^{L}<0$ and $0<z^{H}<\pi(r+\delta)$. This assumption places the $z$ 's sufficiently close to zero that each type will play an inclusive strategy in equilibrium. Since these are the only types, no agents play exclusive strategies $(\lambda(a, a)=\lambda(b, b)=0)$ and as a result $z_{2}=0$ and thus in the market equilibrium there is no conformity. As before, we assume there are $\alpha$ low types and $(1-\alpha)$ high types. We contrast the social welfare when both types play inclusive strategies with the cases in which the types conform either on $a$ or on $b$.

If the two types conform on $a$, meaning that they both build and trade $a$, then, using Lemma 5, the flow of social welfare is simply

$$
W_{(a, a),(a, a)}=1-\delta c,
$$

where the subscript indicates that both types play $(a, a)$ (note that the allocations that emerge when both types play $(a, a)$ are the same as those when both types play $(a, a b))$. Likewise, if they conform on $b$, the flow of welfare is

$$
W_{(b, b),(b, b)}=1+z^{L} \alpha+z^{H}(1-\alpha)-\delta c .
$$

The difference $W_{(b, b),(b, b)}-W_{(a, a),(a, a)}$ is positive if and only if the mean taste $\mu=z^{L} \alpha+z^{H}(1-\alpha)$ is positive.

Computing the flow of welfare for the inclusive cases is more involved because the agents sometimes have a house other than the type they build. After some algebra, we find the flow of social welfare for the inclusive strategy to be

$$
W_{(a, a b)(b, a b)}=1+\alpha\left(\frac{\gamma}{\gamma+\delta}(1-\alpha)\right) z^{L}+(1-\alpha)\left(1-\frac{\gamma}{\gamma+\delta} \alpha\right) z^{H}-\delta c
$$

Comparing the inclusive non-conforming cases with the conforming equilibrium shows that $W_{(a, a),(a, a)}$ is preferred to $W_{(a, a b)(b, a b)}$ if

$$
\alpha\left(\frac{\gamma}{\gamma+\delta}(1-\alpha)\right)\left[-z^{L}\right]>(1-\alpha)\left(1-\frac{\gamma}{\gamma+\delta} \alpha\right) z^{H}
$$


Likewise, conforming on $b$ is preferred to not conforming if

$$
\left(\frac{\gamma}{\gamma+\delta}(1-\alpha)\right) z^{H}>\left(1-\frac{\gamma}{\gamma+\delta}(1-\alpha)\right)\left[-z^{L}\right]
$$

It is easy to show that only one of these conditions can hold at a time so that if conforming on $b$ is preferable to playing an inclusive strategy then it is strictly worse to conform on $a$.

If we take the limit of these expressions as either the trade hazard approaches infinity, or as the depreciation shock approaches zero, these conditions amount to merely saying that conforming on $a$ is preferable if $\mu<0$ and conforming on $b$ is preferable if $\mu>0$. The intuition for this result is clear. If $\gamma$ is very high then an individual agent will not reside in a given house for very long and thus should make a building decision that fits with the preferences of the average agent. If $\delta$ is very low then a given house will remain for very long and will therefore be occupied in equilibrium by the average agent.

The Possibility of Too Much Conformity The previous two examples suggest that there is in general too little conformity in equilibrium. Individuals do not sufficiently internalize trade costs when they decline trades nor do they sufficiently internalize the costs that arise because someone "settles" for a house that isn't a good match. Both forces seem to lead to too little conformity. It is however, possible that these forces could work in opposing directions.

Consider a hybrid example with three types: low, medium and high. Denote these types as $z^{L}, z^{M}$, and $z^{H}$. Assume that $z^{H}$ is high enough to ensure that he follows the $(b, b)$ policy while $z^{L}$ is low enough that he follows an $(a, a b)$ policy. We consider the optimal choice for the middle type. Assume that there are $\mu$ middle agents, $(1-\mu) \alpha$ low agents and $(1-\mu)(1-\alpha)$ high agents.

If the middle types follow strategy $a, a b$ then none of them ever get a $b$ house (the only $b$ builders follow exclusive strategies). If the middle types follow strategy $b, a b$ then, as in the second example, the low and middle types will occasionally hold type $a$ and type $b$ houses. It is not too difficult to show that the difference between the two welfare flows $W_{b, a b}$ and $W_{a, a b}$ is

$$
\begin{aligned}
W_{b, a b}-W_{a, a b}= & \mu \psi_{b, a b}\left[z^{M}+\pi \gamma(1-\mu)(1-\alpha)\right] \\
& +\pi \gamma(1-\mu)(1-\alpha)\left[2\left(1-\psi_{a, a b}\right)(1-\mu) \alpha+\mu \psi_{b, a b}\right] \\
& +(1-\mu) \alpha\left(1-\psi_{a, a b}\right) z^{L} .
\end{aligned}
$$


The first line summarizes the costs and benefits that accrue to the middle agent if they adopt the $b, a b$ policy instead of the $a, a b$ policy. In this case, they reside in the $b$ house for a fraction of time. The fraction they spend in the type $b$ house is $\psi_{b, a b}$. Because there are $(1-\mu)(1-\alpha)$ exclusive $b$ traders, when the middle agents hold the $b$ houses, they reduce the incidence of the trade penalties by $\pi \gamma(1-\mu)(1-\alpha)$. At the same time, holding the $b$ houses reduces their flow utility by $z^{M}$ (recall that the middle types prefer the $a$ house so $z^{M}<0$ ). If these were the only costs and benefits of adopting one policy or another then the critical $z^{M}=-\pi \gamma(1-\mu)(1-\alpha)$ which is simply the build cutoff $z_{2}$ in this example.

The second and third lines of the expression above represent external costs and benefits not internalized by the middle agent. The second line reflects the reduction in trade penalties experienced by both the high and low types. The high types now have a probability $\mu \psi_{b, a b}$ of encountering a middle type who holds a $b$ house in the trade stage. In this case, there will be no trade penalty. In addition, the high types also encounter low types who happen to possess $b$ houses in the trade stage. The number of low types in this state is $(1-\mu) \alpha\left(1-\psi_{a, a b}\right)$. The number 2 reflects the fact that two trade penalties are avoided in this case (one for the low type and one for the high type).

In the equilibrium, $z_{2}=-\pi \gamma(1-\mu)(1-\alpha)<0$. If the last two lines are negative however there will be too much conformity in the sense that the optimal strategy would call for the middle types to play $(a, a b)$ even if they preferred the $b$ house. This would require

$$
z^{L}<-\pi \gamma \frac{1-\alpha}{\alpha}\left[2(1-\mu) \alpha+\mu\left(\frac{\psi_{b, a b}}{1-\psi_{a, a b}}\right)\right] .
$$

Recall that $z^{L}>z_{1}=-\pi(r+\delta+\gamma)$. Thus if

$$
r+\delta+\gamma>\gamma \frac{1-\alpha}{\alpha}\left[2(1-\mu) \alpha+\mu\left(\frac{\psi_{b, a b}}{1-\psi_{a, a b}}\right)\right]
$$

there is a possibility that there could be too much conformity. Solving for $\psi_{b, a b}$ and $1-\psi_{a, a b}$ in terms of $\mu, \alpha, \delta$ and $\gamma$ requires some tedious algebra which we will spare the reader ${ }^{11}$. With these terms, we can rewrite the expression as

$r+\delta+\gamma>\gamma \frac{1-\alpha}{\alpha}\left[2(1-\mu) \alpha+\frac{[\delta(\mu \gamma+\delta)[\delta+\gamma(1-\mu)]+\mu(\mu \gamma+\delta)[\delta+\gamma(1-\mu)(1-\alpha)]]}{[\gamma \delta \mu(1-\mu) \alpha+\mu(\mu \gamma+\delta)(\delta+\gamma(1-\mu)(1-\alpha))]}\right]$

\footnotetext{
${ }^{11}$ Details are available from the authors.
} 
Note that the right hand side goes to zero as $\alpha$ goes to 1 . Therefore for small enough $\alpha$ the above inequality always holds. This is natural. The more agents there are who prefer $a$, the more the social planner wants them to conform. As $\alpha$ increases, we replace the exclusive high types (who prefer $b$ ) with inclusive low types (who prefer $a$ ). As a result, the benefits to conforming on $b$ disappear, while the costs increase.

To summarize, individuals do not internalize the costs and benefits of their actions in the search market. They trade off the costs of living in a house that they don't prefer with the benefits to reducing trade penalties. These costs and benefits exactly balance for the critical agents (those at $z_{2}$ ). The social planner takes all trade costs into account. Also, unlike the individuals in the market equilibrium,the social planner places more weight on the house lived in by the average type. Both considerations typically lead the social planner to choose greater conformity than the market implies. As the last example shows however, there can be too much conformity if these forces work in opposite directions.

A Numerical Illustration The examples above starkly illustrated the forces that cause conformity to be inefficiently low in equilibrium. Those examples considered simple cases with at most three types of agents. Here we consider the equilibrium and optimal level of conformity for a continuum of agents when the taste parameter is distributed normally. Unlike the two-type examples above, the equilibrium here is much too complicated to solve analytically. Instead we numerically solve for the equilibrium and calculate the optimal level of conformity. These examples again demonstrate that there is often too little conformity in equilibrium.

Figure 3 plots the flow of welfare $W$ given by Lemma 5 for various values of the conformity cutoff $z_{2}$. As mentioned above, we assume that the distribution of tastes $F(z)$ is normal with unit variance and with mean $\mu$. We consider three different means: $\mu=0.0, \mu=0.25$ and $\mu=0.5$. The remaining parameters are set as follows: $r=0.02, \delta=0.05$, and $\gamma=0.10$. The discount rate and depreciation rate are roughly in line with their real-world counterparts. The trade hazard rate implies that people move roughly once every ten years. For illustration, we set $\pi=5$ (the building cost $c$ matters neither for equilibrium nor for welfare comparisons). For each mean, we first compute the equilibrium $z_{1}, z_{2}$ and $z_{3}$ and the corresponding match probabilities (the $\lambda$ 's). We then vary the level of conformity by changing $z_{2}$ while holding $z_{1}$ and $z_{3}$ fixed. Each line in Figure 3 corresponds to the flow of welfare for a separate mean. For $\mu=0$, the optimal level 
of conformity and the equilibrium level of conformity are both 0 . For $\mu=0.25$, there is conformity in equilibrium. This is reflected in the negative value of $z_{2}$ (the equilibrium $z_{2}$ is -0.072 ). The optimal level of conformity (holding $z_{1}$ and $z_{3}$ fixed) is even lower: the flow of welfare is maximized at $z_{2}=-0.254$. For $\mu=0.5$, the contrast is even greater. In equilibrium $z_{2}$ is -0.177 while the optimal level of $z_{2}$ is -0.558 .

Figure 4 calculates the optimal choice for all of the cutoffs simultaneously for several different means. The figure compares the optimal cutoffs with the equilibrium cutoffs. The equilibrium and optimal cutoffs are plotted on the vertical axis while the horizontal axis plots the mean of $F(z)$ (again $F$ is normal with a variance of 1 ). Figure 4 shows that for $\mu>0$ there is conformity in equilibrium $\left(z_{2}<0\right)$. Not surprisingly, the equilibrium level of conformity rises with the mean. Notice that the optimal level of conformity rises even faster than the equilibrium level of conformity. Also, as the mean rises, the cutoffs for the exclusive $a$ and $b$ traders both fall. More individuals prefer the $b$ houses and thus there is more and more pressure to decline the $a$ houses in trade.

\subsection{Renting and Leasing}

In this section we extend the model to allow the agents to rent the durable good. Introducing a rental market is a natural extension because it lets people enjoy the durable without having to worry about resale. Intuitively, the option to rent will be more attractive if one has unusual tastes. For instance, luxury or exotic cars are often leased while more mainstream cars are usually bought outright. This is exactly the pattern one would expect given our model. The people who lease exotic cars do not want to conform and thus do not want to participate in the secondary market. In contrast, people who own mainstream cars can easily find buyers if they need to sell. Of course, there are other reasons that attract people to rental markets. For instance, in the rental market for housing, many people rent because they expect to move in the near future (graduate students and untenured faculty for instance). While this is clearly important for the rental market for houses, we abstract from this motive to maintain our focus on conformity.

In the model, the rental market attracts agents with the strongest preferences for $a$ or $b$. In equilibrium this could lead to either more or less conformity as exclusive traders leave the market for owner occupied houses and choose to rent. We focus on the case in which the cost of renting is sufficiently high so that at most one type of house is rented. In this case, an active rental market creates 
even more conformity in the ownership market.

There are two markets in the renting model: the owner-occupied market and the rental market. We assume that when an agent receives the build shock, he now has the option of renting a house rather than building one. If some agents choose to rent, the number of people in the owner-occupied market will be less than 1. Moreover, the distribution of types in the owner-occupied market will differ from the original distribution $F$. Agents who rent pay a constant flow rental cost $q$ for the house they rent. They can leave the rental market and re-enter the build stage at any time. As before, agents in the owner-occupied market follow one of the four policies $(a, a),(a, a b),(b, a b)$ or $(b, b)$. We continue to let $\lambda(x, \tau)$ denote the fraction of owners who possess $x \in\{a, b\}$ but follow the trading rule $\tau \in\{a, b, a b\} .^{12}$

Given the fractions $\lambda(a, a), \lambda(a, a b), \lambda(b, a b)$, and $\lambda(b, b)$, we can construct the values to owning a type $a$ and $b$ house in the owner-occupied market for any agent. These values $V(a), V(b)$ are the same as those in Lemma 2. The continuation value for an agent who receives the build shock is now

$$
B=\max \{R, V(a)-c, V(b)-c\}
$$

where $R$ is the continuation value of renting. Although renters always have the option to return to the build stage, they will never choose to do so in equilibrium. Since renters simply reside in the type of house that suits them best, we can write the value of renting as

$$
R=\max \left\{\frac{u(a)-q}{r}, \frac{u(b)-q}{r}\right\} .
$$

The following proposition shows that for any set of matching probabilities, only the agents with the strongest preferences choose to enter the rental market.

Proposition 4. For fixed $\lambda(a, a), \lambda(a, a b), \lambda(b, a b)$, and $\lambda(b, b)$, there exist two cutoffs $\rho \in\{-\infty\} \cup\left[z_{1}, 0\right]$ and $\eta \in\left[0, z_{3}\right] \cup\{+\infty\}$ such that

1. All agents with taste parameter $z \leq \rho$ choose to rent type $a$ houses.

2. All agents with taste parameter $z \geq \eta$ choose to rent type $b$ houses.

\footnotetext{
${ }^{12}$ Note that the sum of the $\lambda$ 's is still 1 even though the number of owners is no longer the entire population.
} 
3. All agents with taste parameter $z \in(\rho, \eta)$ remain in the owner-occupied market and follow policies prescribed in Proposition 1.

If renting attracts anyone, it attracts people in the tails of the distribution $F$. These agents have strong preferences over which type of house they want to live in and are either paying the trade penalty frequently or have to endure periods in which they live in a house other than the type they prefer. Notice that if $\rho=-\infty$, no one rents $a$. It is also important to realize that the proposition implies that if anyone rents $a$ then all $z<z_{1}$ rent $a$ and so there will be no one who follows the $(a, a)$ policy in the owner occupied market. Similar observations hold for the $b$ rental cutoff.

The cost of renting is $q$ per unit time. If there were free entry and exit into the housing market and if tenants could be instantly and costlessly replaced whenever a vacancy opened up then $q=(r+\delta) c$. This just says that the steady state rental price is equal to the Jorgensonian user cost of capital. In this case, one can show that the rental market completely resolves the matching problem and thus all agents choose to rent. If vacancies are not costless to fill however, the rental price should exceed the user cost. Specifically, we write $q=(r+\delta+m) c>$ $(r+\delta) c$ where $m$ is the rental markup due to matching difficulties. Depending on the magnitude of the markup, the rental market may or may not be active. If the rental market is active, it is possible that only one type of house will be rented. In the discussion below, we make several comparisons between the equilibrium with renting and the equilibrium when there is no possibility of rent. We use a superscript 0 to denote equilibrium variables in the model without rent. Define $m^{a}=\frac{\gamma \pi}{c}\left[\lambda^{0}(b, a b)+\lambda^{0}(b, b)\right], m^{b}=\frac{\gamma \pi}{c}\left[\lambda^{0}(a, a b)+\lambda^{0}(a, a)\right]$ and let $m^{*}=\max \left\{m^{a}, m^{b}\right\}$. (Note that $m^{*} \geq 1 / 2$ ). The following Lemma describes the possibilities that may arise for different values of $m$.

Lemma 6. Let $q=(r+\delta+m) c$ and let $m^{*}$ be defined as above. The following statements are true:

1. If $m=0$ then every agent weakly prefers renting to owning.

2. If $m c>\gamma \pi$ then the rental market is shut down.

3. If $m^{*} c \geq m c>\frac{1}{2} \gamma \pi$ then the rental market must be active in equilibrium but only one type will be rented. ${ }^{13}$

\footnotetext{
${ }^{13}$ Note, if $\gamma \pi>m c>m^{*} c$ it is possible that there is an equilibrium with rent even though the equilibrium without rent is still viable.
} 
4. If $m c<\frac{1}{2} \gamma \pi$ then the rental market must be active in equilibrium and moreover it is possible that both types will be rented.

It is convenient to work with the case in which only one type rents. We therefore assume that $\frac{1}{2} \gamma \pi<m c<m^{*} c$ so that we are in case 3 of the Lemma. To ensure that the equilibrium in the owner-occupied market is still unique, we assume that the distribution of taste parameters has a density $f$ which is symmetric about its mean $\mu$, quasi-concave and single peaked with $f(\mu)<\frac{1}{\pi \gamma} \frac{1}{2}$. We assume that $\mu>0$ so that, by proposition (3), in the absence of the rental market there is a unique equilibrium in which the agents conform on type $b$ houses. In this equilibrium, $z_{2}^{0}<0$ and thus we must have $\lambda^{0}(a, a)>\lambda^{0}(b, b)$.

Now introduce the possibility of renting. By Proposition 4 , associated with the original equilibrium matching probabilities $\lambda(a, a)^{0}, \lambda(b, b)^{0}, \lambda(a, a b)^{0}, \lambda(b, a b)^{0}$ we have cutoffs $\rho^{0}$ and $\eta^{0}$ that identify the agents who wish to enter the rental market. Because $m c<m^{*} c$ (by assumption) Lemma 6 implies that the original equilibrium is no longer valid. Thus, the rental market must be active. In this case, the following proposition says that conformity will increase in the owneroccupied market.

Proposition 5. Let $f$ be symmetric about $\mu>0$, quasi-concave and single peaked with $f(\mu)<\frac{1}{\pi \gamma} \frac{1}{2}$ and assume that $\frac{1}{2} \gamma \pi<m c<m^{*} c$. Then there exists an equilibrium in which only agents who prefer a houses rent. Moreover, in this equilibrium, the owner-occupied market has greater conformity than the equilibrium without the possibility of rent.

Intuitively, the agents who prefer the $a$ houses have the greatest incentive to abandon the owner-occupied market and rent. These agents paid the trade penalty more than half of the time they got the trade shock. The rental market offers them an opportunity to have the type they prefer at a lower effective cost. When the exclusive $a$ traders leave the owner-occupied market, the probability of matching with someone with an $a$ house drops even further. There is more conformity on the type $b$ house and more exclusive $b$ traders. This draws other agents with $z<0$ into the rental market. ${ }^{14}$

\footnotetext{
${ }^{14}$ Note that for lower $m$ there may be equilibria in which both extremes choose to rent. In this case, the owner-occupied market would have no conformity (since there are no exclusive types left). However, it is not necessary to have both types renting for low $m$. In particular, for $m$ in the neighborhood of 0 , there is always an equilibrium in which all agents with $z<0+\varepsilon$ decide to rent which agents with $z>0+\varepsilon$ choose to own (and vice versa).
} 
The function of the rental market in this model is to allow agents to satisfy their tastes for unusual goods without incurring the costs of reselling a good that few others want. Of course, the rental market for housing is not driven primarily by this concern. Casual observation strongly suggests that people who rent do so because they anticipate moving in the near future. At the same time, there are other markets for durable goods where leasing does indeed cater to people with uncommon tastes. The most obvious example of this is the market for luxury automobiles. While leasing has become relatively common in recent years, conventional cars - pickup trucks, Honda Accords, Civics, Ford Escorts, etc.- are rarely leased. In contrast, luxury cars like BMWs, Lexus, Mercedes, etc. are leased frequently.

\section{Discussion and Related Literature}

There are several noteworthy implications and extensions of the model. Perhaps the most glaring simplification in our model is the assumed trading mechanism. Traders are restricted to simple swaps which rules out bargaining with prices. Moreover, traders only get a single match, and if this match does not result in trade they pay a trading penalty and leave the trade stage with the house that they desire. This trading mechanism greatly simplifies the model by making the agents' trading rules independent of the type of house that they possess when they enter the trade stage. While these assumptions are convenient for the analysis they are also highly stylized. Nevertheless, we argue that the insights that emerge from the model will still hold under more realistic assumptions.

First, consider extending the model to allow for price negotiations. There are many trade mechanisms that one could consider (take-it-or-leave-it offers, double auctions etc.). As long as agents preferences (their taste parameters) are private information, none of these mechanisms is ex-post efficient (Myerson and Satterthwaite (1983)). Put differently, some trades with positive surplus will not occur. Thus, while these mechanisms will improve efficiency, they do not fully eliminate the two inefficiencies in the model: Trades will still be rejected too often, and too many people will occupy houses that they do not prefer. Even if we allowed for efficient trading mechanisms (which would require that taste parameters be observable), there would still not be a sufficient degree of conformity. To take a specific example, consider the Nash bargaining solution in which each agent gets half of the surplus in a trade. The efficient amount of conformity requires that agents who build houses fully internalize the costs and benefits that arise 
when this house is transferred to someone else. Because agents get only half of the surplus under Nash bargaining, they do not fully internalize these costs. As a result, even though trade decisions are efficient (taking the build decisions as given, agents trade whenever there is positive joint surplus), the build decisions are still inefficient and there is still too little conformity.

Allowing agents to continue searching after the initial trade is rejected would also leave our basic results intact. Agents would still have an incentive to conform and the level of conformity and the willingness to trade would still be inefficient. In fact, allowing for repeated matches when agents need to sell suggests that there are additional reasons to conform. By owning the type of house that most agents prefer (the majority house), agents reduce their expected sales costs by reducing their time on the market. Owning the minority house implies high costs of trade relative to the other type. We could capture this effect by assuming that the trade penalties were type-specific. If the trade penalties vary inversely with the average willingness to accept that type of house in trade then the incentive to conform is increased.

It is difficult to devise policies that would fully resolve all of the potential inefficiencies in the model. Providing the correct trade incentives, for instance, would likely require taxes or subsidies that are specific to the agents intrinsic preferences. Unlike the incentive to trade, the efficient level of conformity could be achieved with simple government policies. Because our model suggests that diversity in durable goods markets should be discouraged, a tax on unusual houses would improve efficiency.

In addition to the theoretical results, the model generates empirical implications that could conceivably be tested against real world data. For instance, the model predicts that we should observe relatively more conformity in the housing stock in towns with frequent turnover. By the same token, individuals that are likely to move should conform and purchase "typical" houses (or to rent). Tenured professors for example should live in houses with more "character" compared to untenured professors who should own "typical" houses or rent (casual observation suggests that this is indeed the case). Similar arguments hold with other durables. Cars that are leased should, ceteris paribus, be more exotic than cars that are owned.

Our paper is closely related with three separate lines of research. The first is the literature on conformity itself. Perhaps the best-known paper in this literature is Bernheim (1994). In that paper, agents care about both the action they take and their perceived type which in turn depends on their action. Since the agents 
all want to be perceived to be a single common type, their actions reflect both their own ideal action and their desire to be perceived to be of a certain status. When agents care strongly about status, those with intermediate preferences all choose the same action (they conform). Agents with extreme types choose not to conform yet their choices are still distorted somewhat towards the common desired type (Bernheim says that these agents "express their individuality"). The main difference between our environment and Bernheim's is that the desire to conform in our model arises endogenously through resale concerns while in Bernheim's model, agents conform because their preferences place weight on public perceptions of their type. ${ }^{15}$

The second line of research our paper relates to is the literature on durable goods in matching models. Although there are many related papers in this literature, two are particularly noteworthy. Wheaton (1990) considers a search model of housing with two types of occupants (families and singles) and two types of houses (large and small). The focus of his paper is on the optimal level of search intensity. He shows that unless the searcher captures all of the surplus of a trade, there will be too little search effort in the model. Our results on the inefficiency of conformity that flow from trades being declined too often echo Wheaton's finding. Importantly, Wheaton's model is perfectly symmetric and the housing stock is exogenous. Conformity, as we have defined it, deals with the choice of the housing stock and equilibrium asymmetry of types and houses. As a result, he does not address conformity in his model. Smith (2002) considers an environment with many types of agents and many types of perfectly durable goods. Unlike our model, agents are never required to trade. In the model, agents attempt to "trade up" to secure a better durable in the long-run. As a result trade decisions are ultimately governed by the agents individual preferences rather than the preferences of their trading partners. In addition, Smith like Wheaton (1990) makes the supply of

\footnotetext{
${ }^{15}$ There are other important concepts of conformity that have been addressed in the literature. Social norms can be rationalized as equilibria in repeated games. Prominent papers include Akerlof (1980), Kandori (1992), and Okuno-Fujiwara and Postlewaite (1995). In these models agents adhere to social conventions to avoid punishment by other players in the future. Also related is the literature on optimal product diversity. (See among others Spence (1976), Dixit and Stiglitz (1977), Mankiw and Whinston (1987), and Tirole (1993) chapter 7). In these models, consumers have a taste for variety and imperfectly competitive firms make entry decisions. Typically, the equilibrium amount of entry implies a less than optimal level of product diversity (i.e., there is too little entry). In our framework, we highlight the opposite concern. Agents in our model care about resale which endogenously generates a preference against diversity and in equilibrium there is typically too little conformity (too much diversity).
} 
durables exogenous and thus does not focus on equilibrium conformity. ${ }^{16}$

Finally, our model is also related to the literature on liquidity and matching models. (See among others Kiyotaki and Wright (1989) and (1993)). In our model, the equilibrium value of holding a good reflects both its intrinsic utility to the agent and the potential resale value of the good. In matching models of money, the currency (which is durable) is often assumed to be intrinsically worthless (i.e., fiat money). As a result, the equilibrium value of money in these models is due solely to its trade value. Indeed, conformity in our model can be interpreted as arising because of the liquidity value of the durable. The more agents prefer a given type, the higher its resale value and thus more agents will accept it in trade.

\section{Conclusion}

We have shown that a consumer's demand for a durable good is governed not only by his individual preferences but also by the preferences of other market participants. This interdependence of preferences arises in markets for durables because of the inevitable resale of durable goods. If a majority of the people who buy durables desire goods with certain features, then the original owners will choose to buy goods with these features even if they do not like them. The incentive to conform to the majority taste is strongest for long-lived durable goods that are traded frequently. For non-durable goods (goods with a very high depreciation rate) or for durables that are never traded, there is no incentive to conform.

There are two features which lead to conformity in our model. First, because there is a chance that agents will have to sell their house, they care about its resale value. The lower the depreciation rate is, and the more likely it is that they will have to enter the resale market, the more they care about the resale value. In our model, the resale value of the home is determined simply by the likelihood that it will be declined in trade. Second, frictions in the resale market (due to matching) generate the possibility that the house will be purchased by someone with different preferences from the current owner. As a result, the resale value depends on the average preferences of the buyers in the resale market.

In equilibrium, the degree of conformity is typically suboptimal. Surprisingly, there is typically too little conformity. That is, a social planner would prefer that

\footnotetext{
${ }^{16}$ There is also a large literature on the provision and resale of durable goods in market settings. Following Akerlof (1970), much of this literature focuses on how adverse selection problems affect the provision and resale of durables. See Hendel and Lizzeri (1999), (2002), and House and Leahy (2004)) for recent contributions.
} 
agents think about the majority more than they actually do. By not conforming, agents impose two negative externalities on members of the majority. First, people with the majority taste incur greater search costs because of product diversity. Second, some members of the majority with moderate preferences settle for the type they do not prefer. Inefficiently low conformity arises because the original builders do not fully internalize these external costs.

Finally, we show that rental markets are likely to arise in environments in which there is already a high degree of conformity. In this case, the agents with the minority preferences leave and join the rental market and conformity in the owner-occupied market increases.

\section{References}

Akerlof, George. 1970. "The Market for 'Lemons': Quality Uncertainty and the Market Mechanism." Quarterly Journal of Economics 84, August, pp.488500 .

Akerlof, George. 1980. "A Theory of Social Custom, of Which Unemployment May be One Consequence." Quarterly Journal of Economics 94(4), June, pp. 749-775.

Bernheim, B. Douglas. 1994. "A Theory of Conformity." Journal of Political Economy 102(5), October, pp. 841-877.

Dixit, Avinash and Stiglitz, Joseph. 1977. "Monopolistic Competition and Optimum Product Diversity." American Economic Review 67(3), June, pp. 297-308.

Hendel, Igal and Lizzeri, Alessandro. 1999. "Adverse Selection in Durable Goods Markets." American Economic Review. 89, December, pp. 1097-1115.

Hendel, Igal, and Lizzeri, Alessandro. "The Role of Leasing under Adverse Selection." Journal of Political Economy 89 (February 2002): 1097-1115.

House, Christopher L. and Leahy, John V. 2004. "An $s S$ Model with Adverse Selection." Journal of Political Economy. 112(3), pp. 581-613.

Kandori, Michihiro. 1992. "Social Norms and Community Enforcement." Review of Economic Studies 59(1), January, pp. 63-80. 
Kiyotaki, Nobuhiro and Wright, Randall. 1989. "On Money as a Medium of Exchange." Journal of Political Economy, 97(4), pp. 927-954.

Kiyotaki, Nobuhiro and Wright, Randall. 1993. "A Search Theoretic Approach to Monetary Economics." American Economic Review, 83(1), pp. 63-77.

Mankiw, N. Gregory and Whinston, Michael. 1986. "Free Entry and Social Efficiency." Rand Journal of Economics 17(1), Spring, pp. 48-58.

Okuno-Fujiwara, Masahiro and Postlewaite, Andrew. 1995. "Social Norms and Random Matching Games." Games and Economic Behavior 9, pp. 79-109.

Smith, Lones. 2002. "A Model of Exchange where Beauty is in the Eye of the Beholder." working paper, University of Michigan.

Spence, Michael. 1976. "Product Selection, Fixed Costs, and Monopolistic Competition." Review of Economic Studies 43(2), June, pp. 217-235.

Tirole, Jean. The Theory of Industrial Organization. The MIT Press. Cambridge, Massachusetts. 1993.

Wheaton, William. 1990. "Vacancy, Search, and Prices in a Housing Market Matching Model." Journal of Political Economy 98(6), December, pp. 12701292. 


\section{Appendix: Proofs of the Propositions}

Lemma 1 If an agent builds type $x$, then he accepts type $x$ in trade.

Proof. The proof is trivial and is identical for $a$ and $b$. If the agent builds type $a$ then $V(a) \geq V(b)$. Then if he is offered $a$ in trade, he gets $V(a)$ if he accepts the offer and $V(a)-\pi$ if he declines the offer.

Lemma 2 Given matching probabilities $\lambda(x, \tau)$ for $x \in\{a, b\}$ and $\tau \in\{a, b, a b\}$ with $\sum_{\tau \in\{a, b, a b\}} \sum_{y \in\{a, b\}} \lambda(y, \tau)=$ 1 there exist unique values $V(a), V(b), T(a), T(b)$, and $B$ satisfying (1), (2), and (3).

Proof. The proof follows from standard dynamic programming techniques.

Lemma 3 Let $z_{1}, z_{2}$ and $z_{3}$ be given. Define

$$
r_{a}=\frac{\delta+\gamma F\left(z_{1}\right)}{F\left(z_{3}\right)-F\left(z_{2}\right)}, r_{b}=\frac{\delta+\gamma\left[1-F\left(z_{3}\right)\right]}{F\left(z_{2}\right)-F\left(z_{1}\right)}
$$

then the steady state values of $P_{a, a b}(a), P_{a, a b}(b), P_{b, a b}(a)$, and $P_{b, a b}(b)$ are given by

$$
\begin{aligned}
& P_{a, a b}(b)=\frac{\gamma r_{b}}{\gamma r_{a}+\gamma r_{b}+r_{a} r_{b}}\left(F\left(z_{2}\right)-F\left(z_{1}\right)\right), P_{a, a b}(a)=\frac{\gamma r_{a}+r_{a} r_{b}}{\gamma r_{a}+\gamma r_{b}+r_{a} r_{b}}\left(F\left(z_{2}\right)-F\left(z_{1}\right)\right) \\
& P_{b, a b}(a)=\frac{\gamma r_{a}}{\gamma r_{a}+\gamma r_{b}+r_{a} r_{b}}\left(F\left(z_{3}\right)-F\left(z_{2}\right)\right), P_{b, a b}(b)=\frac{\gamma r_{b}+r_{a} r_{b}}{\gamma r_{a}+\gamma r_{b}+r_{a} r_{b}}\left(F\left(z_{3}\right)-F\left(z_{2}\right)\right)
\end{aligned}
$$

and the steady state matching probabilities are

$$
\begin{gathered}
\lambda(a, a)=F\left(z_{1}\right), \lambda(b, b)=1-F\left(z_{3}\right) \\
\lambda(a, a b)=P_{a, a b}(a)+P_{b, a b}(a), \lambda(b, a b)=P_{a, a b}(b)+P_{b, a b}(b)
\end{gathered}
$$

Proof. Because in the steady state, $P_{a, a b}(a)$ is constant, we had,

$$
\gamma P_{a, a b}(a) P_{b, a b}(b)=P_{a, a b}(b)\left[\gamma\left(P_{b, a b}(a)+P_{a, a}(a)\right)+\delta\right]
$$

This implies:

$$
\begin{gathered}
\gamma P_{a, a b}(a) P_{b, a b}(b)-\gamma P_{a, a b}(b) P_{b, a b}(a)=\gamma P_{a, a b}(b) P_{a, a}(a)+P_{a, a b}(b) \delta \\
\gamma\left[\frac{P_{a, a b}(a) P_{b, a b}(b)}{P_{a, a b}(b) P_{b, a b}(a)}-1\right]=\left(\gamma P_{a, a}(a)+\delta\right) \frac{1}{P_{b, a b}(a)}
\end{gathered}
$$

Define $B$ as:

$$
B=\gamma\left[\frac{P_{b, a b}(b)}{P_{b, a b}(a)} \frac{P_{a, a b}(a)}{P_{a, a b}(b)}-1\right]
$$

then we can write:

$$
P_{b, a b}(a)=\frac{\delta+\gamma P_{a, a}(a)}{B}
$$

Similar arguments for the $\{b, a b\}$ agents give

$$
P_{a, a b}(b)=\frac{\delta+\gamma P_{b, b}(b)}{B}
$$

The total number of people who follow strategy $\{a, a b\}$ is $F\left(z_{2}\right)-F\left(z_{1}\right)$, thus,

$$
P_{a, a b}(a)=F\left(z_{2}\right)-F\left(z_{1}\right)-\frac{\delta+\gamma P_{b, b}(b)}{B} .
$$

Similarly, for $\{b, a b\}$ agents,

$$
P_{b, a b}(b)=F\left(z_{3}\right)-F\left(z_{2}\right)-\frac{\delta+\gamma P_{a, a}(a)}{B}
$$


We can now form the ratios $\frac{P_{a, a b}(a)}{P_{a, a b}(b)}$ and $\frac{P_{b, a b}(b)}{P_{b, a b}(a)}$ as

Recalling the definition of $B$

$$
\begin{aligned}
& \frac{P_{a, a b}(a)}{P_{a, a b}(b)}=\frac{\left[F\left(z_{2}\right)-F\left(z_{1}\right)\right] B}{\delta+\gamma P_{b, b}(b)}-1 \\
& \frac{P_{b, a b}(b)}{P_{b, a b}(a)}=\frac{\left[F\left(z_{3}\right)-F\left(z_{2}\right)\right] B}{\delta+\gamma P_{a, a}(a)}-1
\end{aligned}
$$

$$
\begin{aligned}
B & =\gamma\left[\frac{P_{b, a b}(b)}{P_{b, a b}(a)} \frac{P_{a, a b}(a)}{P_{a, a b}(b)}-1\right] \\
& =\gamma\left[\left(\frac{\left[F\left(z_{3}\right)-F\left(z_{2}\right)\right] B}{\delta+\gamma P_{a, a}(a)}-1\right)\left(\frac{\left[F\left(z_{2}\right)-F\left(z_{1}\right)\right] B}{\delta+\gamma P_{b, b}(b)}-1\right)-1\right]
\end{aligned}
$$

we get

$$
B=\frac{\delta+\gamma P_{a, a}(a)}{\left[F\left(z_{3}\right)-F\left(z_{2}\right)\right]}+\frac{\delta+\gamma P_{b, b}(b)}{\left[F\left(z_{2}\right)-F\left(z_{1}\right)\right]}+\frac{\left(\delta+\gamma P_{a, a}(a)\right)\left(\delta+\gamma P_{b, b}(b)\right)}{\gamma\left[F\left(z_{2}\right)-F\left(z_{1}\right)\right]\left[F\left(z_{3}\right)-F\left(z_{2}\right)\right]}
$$

Use the fact that $P_{a, a}(a)=F\left(z_{1}\right)$ and $P_{b, b}(b)=1-F\left(z_{3}\right)$ as well as $r_{a}$ and $r_{b}$ as defined in the statement of the Lemma, we can express $B$ as

$$
B=r_{a}+r_{b}+\frac{r_{a} r_{b}}{\gamma}
$$

We can now solve for $P_{a, a b}(a), P_{a, a b}(b), P_{b, a b}(a)$ and $P_{b, a b}(b)$.

Finally, by construction,

$$
\begin{aligned}
P_{a, a b}(b) & =\frac{\delta+\gamma P_{b, b}(b)}{B}=\frac{r_{b}\left(F\left(z_{2}\right)-F\left(z_{1}\right)\right)}{B} \\
& =\frac{\gamma r_{b}}{\gamma r_{a}+\gamma r_{b}+r_{a} r_{b}}\left(F\left(z_{2}\right)-F\left(z_{1}\right)\right) \\
P_{a, a b}(a) & =\frac{\gamma r_{a}+r_{a} r_{b}}{\gamma r_{a}+\gamma r_{b}+r_{a} r_{b}}\left(F\left(z_{2}\right)-F\left(z_{1}\right)\right) \\
P_{b, a b}(a) & =\frac{\gamma r_{a}}{\gamma r_{a}+\gamma r_{b}+r_{a} r_{b}}\left(F\left(z_{3}\right)-F\left(z_{2}\right)\right) \\
P_{b, a b}(b) & =\frac{\gamma r_{b}+r_{a} r_{b}}{\gamma r_{a}+\gamma r_{b}+r_{a} r_{b}}\left(F\left(z_{3}\right)-F\left(z_{2}\right)\right)
\end{aligned}
$$

$$
\begin{aligned}
& \lambda(a, a b)=P_{a, a b}(a)+P_{b, a b}(a) . \\
& \lambda(b, a b)=P_{a, a b}(b)+P_{b, a b}(b) .
\end{aligned}
$$

Lemma 4 The mappings $L_{a, a}$ and $L_{b, b}$ defined by (5) and (6) each have at least one fixed point.

Proof. Consider $\lambda$ and $\lambda^{\prime}$ both in $[0,1]$ with $\lambda^{\prime} \geq \lambda$. It is straight forward to show that $L_{a, a}\left(\lambda^{\prime}\right) \geq$ $L_{a, a}(\lambda)$. Existence then follows by Tarski's fixed point theorem. The proof for $L_{b, b}$ is identical.

Lemma 5 For any given cutoffs $z_{1}, z_{2}$ and $z_{3}$, let $P_{a, a b}(a), P_{b, a b}(a), P_{a, a b}(b)$ and $P_{b, a b}(b)$ be given as in Lemma 3 and define

$$
\psi_{a, a b}=\left(\frac{P_{a, a b}(a)}{F\left(z_{2}\right)-F\left(z_{1}\right)}\right) \text { and } \psi_{b, a b}=\left(\frac{P_{b, a b}(b)}{F\left(z_{3}\right)-F\left(z_{2}\right)}\right) .
$$

Then, the flow of social welfare is

$$
\begin{aligned}
W\left(z_{1}, z_{2}, z_{3}\right) & =\{1-\pi \gamma[\lambda(b, b)+\lambda(b, a b)]\} F\left(z_{1}\right) \\
& +\int_{z_{1}}^{z_{2}}\left\{\psi_{a, a b}[1-\pi \gamma \lambda(b, b)]+\left(1-\psi_{a, a b}\right)[1+z-\pi \gamma \lambda(a, a)]\right\} d F(z) \\
& +\int_{z_{2}}^{z_{3}}\left\{\left(1-\psi_{b, a b}\right)[1-\pi \gamma \lambda(b, b)]+\psi_{b, a b}[1+z-\pi \gamma \lambda(a, a)]\right\} d F(z) \\
& +\int_{z_{3}}^{\infty}\{1+z-\pi \gamma[\lambda(a, a)+\lambda(a, a b)]\} d F(z)-\delta c .
\end{aligned}
$$


Proof. The probability that an agent who follows strategy $(a, a)$ incurs the trade penalty is

$$
\gamma[\lambda(b, b)+\lambda(b, a b)]
$$

and the probability for a $(b, b)$ agent is

$$
\gamma[\lambda(a, a)+\lambda(a, a b)]
$$

The flow utility for the $(a, a)$ 's is

$$
\int_{-\infty}^{z_{1}}\{1-\delta c-\pi \gamma[\lambda(b, b)+\lambda(b, a b)]\} d F(z)
$$

and for the $(b, b)$ 's is

$$
\int_{z_{3}}^{\infty}\{1+z-\delta c-\pi \gamma[\lambda(a, a)+\lambda(a, a b)]\} d F(z)
$$

The agents who follow inclusive strategies are somewhat more involved. Begin by considering the flow utility of a particular agent of type $z$. Take any $z \in\left[z_{1}, z_{2}\right]$. These agents play $(a, a b)$. If the agent has $a$ in inventory the probability of incurring the trade penalty is $\gamma \lambda(b, b)$, if they have $b$ in inventory the probability of incurring the trade penalty is, $\gamma \lambda(a, a)$. The probability of incurring the building cost (the same in either case) is $\delta$. The fraction of $(a, a b)$ agents that hold the house they build $(a)$ is

$$
\frac{P_{a, a b}(a)}{F\left(z_{2}\right)-F\left(z_{1}\right)}
$$

The flow utility from the agents with this $z$ is thus

$$
\left(\frac{P_{a, a b}(a)}{F\left(z_{2}\right)-F\left(z_{1}\right)}\right)[1-\pi \gamma \lambda(b, b)]+\left(1-\frac{P_{a, a b}(a)}{F\left(z_{2}\right)-F\left(z_{1}\right)}\right)[1+z-\pi \gamma \lambda(a, a)]-\delta c .
$$

The flow utility for the entire group of $(a, a b)$ agents is

$$
\int_{z_{1}}^{z_{2}}\left\{\left(\frac{P_{a, a b}(a)}{F\left(z_{2}\right)-F\left(z_{1}\right)}\right)[1-\pi \gamma \lambda(b, b)]+\left(1-\frac{P_{a, a b}(a)}{F\left(z_{2}\right)-F\left(z_{1}\right)}\right)[1+z-\pi \gamma \lambda(a, a)]-\delta c\right\} d F(z)
$$

Similar arguments give the flow utility for the $(b, a b)$ traders as

$$
\int_{z_{2}}^{z_{3}}\left\{\left(1-\frac{P_{b, a b}(b)}{F\left(z_{3}\right)-F\left(z_{2}\right)}\right)[1-\pi \gamma \lambda(b, b)]+\left(\frac{P_{b, a b}(b)}{F\left(z_{3}\right)-F\left(z_{2}\right)}\right)[1+z-\pi \gamma \lambda(a, a)]-\delta c\right\} d F(z)
$$

Total welfare $(W)$ is the sum of the flow utility of the four types which can be rearranged to get

$$
\begin{aligned}
W= & 1+\left(\frac{P_{a, a b}(b)}{F\left(z_{2}\right)-F\left(z_{1}\right)}\right) \int_{z_{1}}^{z_{2}} z d F(z)+\frac{P_{b, a b}(b)}{F\left(z_{3}\right)-F\left(z_{2}\right)} \int_{z_{2}}^{z_{3}} z d F(z)+\int_{z_{3}}^{\infty} z d F(z)-\delta c \\
& -2 \pi \gamma\left\{F\left(z_{1}\right)\left[1-F\left(z_{3}\right)\right]+\left[P_{a, a b}(b)+P_{b, a b}(b)\right] F\left(z_{1}\right)+\left[P_{a, a b}(a)+P_{b, a b}(a)\right]\left[1-F\left(z_{3}\right)\right]\right\}
\end{aligned}
$$

Lemma 6 Let $q=(r+\delta+m) c, m^{a}=\frac{\gamma \pi}{c}\left[\lambda^{0}(b, a b)+\lambda^{0}(b, b)\right], m^{b}=\frac{\gamma \pi}{c}\left[\lambda^{0}(a, a b)+\lambda^{0}(a, a)\right]$ and $m^{*}=$ $\max \left\{m^{a}, m^{b}\right\}$. The following statements are true:

1. If $m=0$ then every agent weakly prefers renting to owning.

2. If $m c>\gamma \pi$ then the rental market is shut down.

3. If $m^{*} c \geq m c>\frac{1}{2} \gamma \pi$ then the rental market must be active in equilibrium but only one type will be rented.

4. If $m c<\frac{1}{2} \gamma \pi$ then the rental market must be active in equilibrium. 
Proof. Type $(a, a)$ will prefer renting if

$$
q \leq \gamma \pi[\lambda(b, a b)+\lambda(b, b)]+(r+\delta) c
$$

type $(b, b)$ prefers renting if

$$
q \leq \gamma \pi[\lambda(a, a b)+\lambda(a, a)]+(r+\delta) c
$$

If $m=0$ then the inequalities hold for both $(a, a)$ and $(b, b)$ (the inequality is strict as long as $\lambda(b, a b)+$ $\lambda(b, b)$ and $\lambda(a, a b)+\lambda(a, a)$ are each greater than 0$)$. This establishes 1.

Since neither $\lambda(b, a b)+\lambda(b, b)$ nor $\lambda(a, a b)+\lambda(a, a)$ can be greater than 1 , the inequality is reversed for $m c>\gamma \pi$. This establishes 2 .

Note that if both $(a, a)$ and $(b, b)$ and prefered renting then, adding the expressions above, it must be the case that

$$
m c \leq \frac{\gamma \pi}{2}
$$

which is impossible if $m c>\frac{\gamma \pi}{2}$. Thus if $m c>\frac{\gamma \pi}{2}$ only one type can rent. Moreover, if $m c<m^{*} c$ it is clear that the original equilibrium cannot survive as one or the other exclusive types will deviate to the rental market. This establishes 3 .

Finally, observe that in any equilibrium, either $\lambda(b, a b)+\lambda(b, b) \leq \frac{1}{2}$ or $\lambda(a, a b)+\lambda(a, a) \leq \frac{1}{2}$. Thus if $m c<\frac{1}{2} \gamma \pi$ either the $(a, a)$ types rent or the $(b, b)$ types rent (or both). This establishes 4 .

Lemma 7 If a density function $f(z)$ with mean $\mu$ satisfies the following conditions:

1. Symmetry $(S)$ : for any $x, f(\mu+x)=f(\mu-x)$, and $F(\mu+x)=1-F(\mu-x)$.

2. Quasi-Concave $(Q C)$ : for any fixed $x$, the set $\{y: f(y) \geq f(x)\}$ is convex

then for any $z<z^{\prime}, F(z) \geq 1-F\left(z^{\prime}\right) \Leftrightarrow f(z) \geq f\left(z^{\prime}\right)$.

Proof. Define $x$ and $x^{\prime}$ implicitly as $z=\mu+x$ and $z^{\prime}=\mu+x^{\prime}$. Since $z^{\prime}>z, x^{\prime}>x$. Clearly $z$ is either greater than or less than the mean $\mu$.

1. Suppose $z \leq \mu$ (so that $x \leq 0)$ then,

(a) $F(z) \geq 1-F\left(z^{\prime}\right) \Rightarrow F(\mu+x) \geq 1-F\left(\mu+x^{\prime}\right) \Rightarrow$ (by $\left.S\right) \Rightarrow 1-F(\mu-x) \geq 1-F\left(\mu+x^{\prime}\right) \Rightarrow$ $F\left(\mu+x^{\prime}\right) \geq F(\mu-x) \Rightarrow x^{\prime}>-x \geq 0$ and $0 \geq x>-x^{\prime}$. We now have $f\left(z^{\prime}\right)=f\left(\mu+x^{\prime}\right)=$ $f\left(\mu-x^{\prime}\right) \leq f(y)$ for all $y \in\left[\mu-x^{\prime}, \mu+x^{\prime}\right]$ (by $Q C$ ). Since $z=\mu+x>\mu-x^{\prime}$ and $z<z^{\prime}=\mu+x^{\prime}$, $z$ is in this interval. Thus $f(z) \geq f\left(z^{\prime}\right)$.

(b) $F(z) \leq 1-F\left(z^{\prime}\right) \Rightarrow F(\mu+x) \leq 1-F\left(\mu+x^{\prime}\right) \Rightarrow$ (by $\left.S\right) \Rightarrow F(\mu+x) \leq F\left(\mu-x^{\prime}\right) \Rightarrow x^{\prime}<$ $-x \Rightarrow x<-x^{\prime} \Rightarrow f(z)=f(\mu+x)=f(\mu-x) \leq f(y)$ for all $y \in[\mu+x, \mu-x]$ (by $Q C$ ) (recall that $x \leq 0)$. Since $z^{\prime}=\mu+x^{\prime}<\mu-x$ and $z^{\prime}>z=\mu+x, z^{\prime}$ is in this interval. Thus $f\left(z^{\prime}\right) \geq f(z)$.

Thus if $z \leq \mu, F(z) \geq 1-F\left(z^{\prime}\right) \Leftrightarrow f(z) \geq f\left(z^{\prime}\right)$.

2. Suppose that $z \geq \mu$ so that $z^{\prime} \geq \mu$. Because $z^{\prime}>z \geq \mu$ and $F$ is symmetric, $F(z) \geq \frac{1}{2} \geq 1-F\left(z^{\prime}\right)$. Moreover, $f(y) \geq f\left(z^{\prime}\right)$ for all $y \in\left[\mu-x^{\prime}, \mu+x^{\prime}\right]$. Since $z \in\left[\mu, z^{\prime}\right] \subset\left[\mu-x^{\prime}, \mu+x^{\prime}\right]$ we must have $f(z) \geq f\left(z^{\prime}\right)$.

Thus $z \geq \mu \Rightarrow F(z) \geq 1-F\left(z^{\prime}\right)$ and $f(z) \geq f\left(z^{\prime}\right)$.

Lemma 8 For the equilibrium without the possibility of rent, the following statements are true:

1. If $\mu>0$ then $F\left(z_{3}\right)-F\left(z_{2}\right)>F\left(z_{2}\right)-F\left(z_{1}\right)$ and $\lambda(b, b)+\lambda(b, a b)>\frac{1}{2}>\lambda(a, a)+\lambda(a, a b)$.

2. If $\mu<0$ then $F\left(z_{3}\right)-F\left(z_{2}\right)<F\left(z_{2}\right)-F\left(z_{1}\right)$ and $\lambda(b, b)+\lambda(b, a b)<\frac{1}{2}<\lambda(a, a)+\lambda(a, a b)$. 
Proof. We prove the Lemma for the case $\mu>0$ (the proof for $\mu<0$ is identical). Note that $\mu>0$ implies that $\lambda(a, a)<\lambda(b, b)$ and thus $z_{2}<0$. Recall that $z_{2}=z_{1}+z_{3}$ and that $z_{1}<0<z_{3}$. Since $z_{2}<0$ it must be the case that $\left|z_{1}\right|>z_{3}$. However, the distance $z_{2}-z_{1}=z_{3}$ while $z_{3}-z_{2}=\left|z_{1}\right|$. Thus,

$$
z_{3}-z_{2}>z_{2}-z_{1}
$$

Since $f$ is single-peaked, $f$ is increasing in $z$ for $z<\mu$ and decreasing in $z$ for $z>\mu$. We will show that for any $x>0, f\left(z_{2}+x\right)>f\left(z_{2}-x\right)$. Fix $x>0$. If $z_{2}+x>\mu$ then, $f\left(z_{2}+x\right)>f(\mu+x)$ (because $\left.z_{2}+x<\mu+x\right)$. In addition, $f\left(z_{2}-x\right)<f(\mu-x)$. Since $f(\mu+x)=f(\mu-x)$ we have $f\left(z_{2}+x\right)>f\left(z_{2}-x\right)$. If $z_{2}+x<\mu$ then $f\left(z_{2}+x\right)>f\left(z_{2}-x\right)$ because $f$ is increasing throughout this range.

Now consider $F\left(z_{3}\right)-F\left(z_{2}\right)$ relative to $F\left(z_{2}\right)-F\left(z_{1}\right)$. By assumption $\mu>0$. Make the following change of variable: let $y=2 z_{2}-z$ and write these as

$$
\begin{aligned}
\int_{z_{1}}^{z_{2}} f(z) d z & =-\int_{z_{2}+\left(z_{2}-z_{1}\right)}^{z_{2}} f\left(2 z_{2}-y\right) d y=\int_{z_{2}}^{z_{2}+\left(z_{2}-z_{1}\right)} f\left(2 z_{2}-y\right) d y \\
& \leq \int_{z_{2}}^{z_{2}+\left(z_{2}-z_{1}\right)} f(y) d y<\int_{z_{2}}^{z_{3}} f(y) d y
\end{aligned}
$$

Here the first inequality (the third line) follows from the fact that $f\left(z_{2}+x\right)>f\left(z_{2}-x\right)$. To see this, let $x=y-z_{2}$ which is positive. The last inequality follows from the fact that $z_{3}-z_{2}>z_{2}-z_{1}$.

With $F\left(z_{3}\right)-F\left(z_{2}\right)>F\left(z_{2}\right)-F\left(z_{1}\right)$, we can use the fomula's in Lemma (3), to get the ratio of $r_{b}$ to $r_{a}$ as

$$
\frac{r_{b}}{r_{a}}=\left(\frac{F\left(z_{3}\right)-F\left(z_{2}\right)}{F\left(z_{2}\right)-F\left(z_{1}\right)}\right)\left(\frac{\delta+\gamma\left[1-F\left(z_{3}\right)\right]}{\delta+\gamma F\left(z_{1}\right)}\right)=\left(\frac{F\left(z_{3}\right)-F\left(z_{2}\right)}{F\left(z_{2}\right)-F\left(z_{1}\right)}\right)\left(\frac{\delta+\gamma \lambda(b, b)}{\delta+\gamma \lambda(a, a)}\right)>1
$$

Thus $r_{b}>r_{a}$. In the equilibrium, $\lambda(a, a b)=P_{a, a b}(a)+P_{b, a b}(a)$ and $\lambda(b, a b)=P_{a, a b}(b)+P_{b, a b}(b)$. Again appealing to Lemma (3) gives

$$
\begin{aligned}
& \lambda(b, b)+\lambda(b, a b)=\lambda(b, b)+\frac{r_{a} r_{b}}{\gamma r_{a}+\gamma r_{b}+r_{a} r_{b}}\left(F\left(z_{3}\right)-F\left(z_{2}\right)\right)+\frac{\gamma r_{b}}{\gamma r_{a}+\gamma r_{b}+r_{a} r_{b}}\left(F\left(z_{3}\right)-F\left(z_{1}\right)\right) \\
& \lambda(a, a)+\lambda(a, a b)=\lambda(a, a)+\frac{r_{a} r_{b}}{\gamma r_{a}+\gamma r_{b}+r_{a} r_{b}}\left(F\left(z_{2}\right)-F\left(z_{1}\right)\right)+\frac{\gamma r_{a}}{\gamma r_{a}+\gamma r_{b}+r_{a} r_{b}}\left(F\left(z_{3}\right)-F\left(z_{1}\right)\right)
\end{aligned}
$$

Subtracting the second expression from the first gives the difference $D=\lambda(b, b)+\lambda(b, a b)-[\lambda(a, a)+\lambda(a, a b)]$ as

$D=\lambda(b, b)-\lambda(a, a)+\frac{r_{a} r_{b}}{\gamma r_{a}+\gamma r_{b}+r_{a} r_{b}}\left[\left(F\left(z_{3}\right)-F\left(z_{2}\right)\right)-\left(F\left(z_{2}\right)-F\left(z_{1}\right)\right)\right]+\frac{\gamma r_{b}-\gamma r_{a}}{\gamma r_{a}+\gamma r_{b}+r_{a} r_{b}}\left(F\left(z_{3}\right)-F\left(z_{1}\right)\right)$

Each of these terms is positive which completes the proof.

Proposition 1 Given non-negative $\lambda(a, a), \lambda(b, b), \lambda(a, a b)$, and $\lambda(b, a b)$ summing to 1 , define $z_{1}, z_{2}$, and $z_{3}$ as follows:

$$
\begin{gathered}
z_{1}=-\pi\{r+\delta+\gamma[1-\lambda(a, a)]\} \\
z_{2}=\gamma \pi[\lambda(a, a)-\lambda(b, b)] \\
z_{3}=\pi\{r+\delta+\gamma[1-\lambda(b, b)]\}
\end{gathered}
$$

Then, for any $z<z_{1}$ the optimal strategy is $(a, a)$; for $z_{1}<z<z_{2}$ the optimal strategy is $(a, a b)$; for any $z_{2}<z<z_{3}$ the optimal strategy is $(b, a b)$ and for $z>z_{3}$ the optimal strategy is $(b, b)$.

Proof. We focus on the continuation value when an agent receives the build shock. At this point, an agent chooses the type of house to build and formulates a contingency plan. Our strategy for the proof is to calculate the building continuation value for each possible strategy. Agents must follow one of the following four strategies $\{a, a\},\{a, a b\},\{b, a b\}$, and $\{b, b\}$. The optimal strategy is the one with the highest value in the building stage. By construction, the dynamic programming problem is dynamically consistent. Thus if it is optimal to follow strategy $\tau$ in one state, it is optimal to follow it in any other state.

Suppose that $z$ follows strategy $\{a, a\}$. We write $v_{a, a}($.$) and \tau_{a, a}($.$) to denote the value of following this$ strategy and the expected value of receiving the trade shock. Note these strategies may not be optimal (i.e. $v_{a, a}(x) \leq V(x)$ and $\left.\tau_{a, a}(x) \leq T(x)\right)$. We consider each strategy in turn. 
1. Strategy $\{a, a\}$. If $z$ follows $\{a, a\}$ then he always rejects $b$ in the trade stage $\tau_{a, a}(b)$ is irrelevant. The trade value $\tau_{a, a}(a)$ is

$$
\tau_{a, a}(a)=v_{a, a}(a)-\pi[\lambda(b, a b)+\lambda(b, b)]
$$

The consumption stage value is then,

$$
v_{a, a}(a)=\frac{1-\delta c-\gamma \pi[\lambda(b, a b)+\lambda(b, b)]}{r}
$$

2. Strategy $\{a, a b\}$. When $z$ accepts either $a$ or $b$ in the trading stage, we must calculate both the trade value of $a$ and $b$. Similarly, we must also consider the consumption value of both $a$ and $b$. We begin with the value functions in the trading stages. The trade value of possessing $a$ is

$$
\tau_{a, a b}(a)=v_{a, a b}(a)+\lambda(b, a b)\left[v_{a, a b}(b)-v_{a, a b}(a)\right]-\pi \lambda(b, b)
$$

The trade value of possessing $b$ is

$$
\tau_{a, a b}(b)=v_{a, a b}(a)+[\lambda(b, b)+\lambda(b, a b)]\left[v_{a, a b}(b)-v_{a, a b}(a)\right]-\pi \lambda(a, a)
$$

The consumption value for $a$ satisfies

$$
r v_{a, a b}(a)=1-\delta c+\gamma\left\{\lambda(b, a b)\left[v_{a, a b}(b)-v_{a, a b}(a)\right]-\pi \lambda(b, b)\right\}
$$

while the consumption value for $b$ satisfies

$$
\begin{aligned}
r v_{a, a b}(b)= & 1+z+\delta\left[v_{a, a b}(a)-v_{a, a b}(b)-c\right] \\
& +\gamma\left\{v_{a, a b}(a)-v_{a, a b}(b)+[\lambda(b, b)+\lambda(b, a b)]\left[v_{a, a b}(b)-v_{a, a b}(a)\right]-\pi \lambda(a, a)\right\}
\end{aligned}
$$

or,

$$
\begin{aligned}
r v_{a, a b}(b)= & 1+z-\delta c-\delta\left[v_{a, a b}(b)-v_{a, a b}(a)\right]+\gamma\left\{[\lambda(b, b)+\lambda(b, a b)]\left[v_{a, a b}(b)-v_{a, a b}(a)\right]-\pi \lambda(a, a)\right\} \\
& -\gamma\left[v_{a, a b}(b)-v_{a, a b}(a)\right]
\end{aligned}
$$

Subtracting (8) gives

$$
v_{a, a b}(b)-v_{a, a b}(a)=\frac{z+\gamma \pi[\lambda(b, b)-\lambda(a, a)]}{r+\delta+\gamma[1-\lambda(b, b)]}
$$

Plugging this back into (8) we get the consumption value $v_{a, a b}(a)$.

$$
r v_{a, a b}(a)=1+\gamma\left\{\lambda(b, a b)\left[\frac{z+\gamma \pi[\lambda(b, b)-\lambda(a, a)]}{r+\delta+\gamma[1-\lambda(b, b)]}\right]-\pi \lambda(b, b)\right\}-\delta c .
$$

To compare the two strategies $\{a, a b\}$ and $\{a, a\}$, consider an agent who receives the build shock. If he decides to follow $\{a, a b\}$ then his payoff is $v_{a, a b}(a)-c$ while if he follows $\{a, a\}$ his payoff is $v_{a, a}(a)-c$. The agent will thus prefer $\{a, a b\}$ to $\{a, a\}$ if $v_{a, a b}(a)>v_{a, a}(a) \Leftrightarrow$

$$
1+\gamma\left\{\lambda(b, a b)\left[\frac{z+\gamma \pi[\lambda(b, b)-\lambda(a, a)]}{r+\delta+\gamma[1-\lambda(b, b)]}\right]-\pi \lambda(b, b)\right\}-\delta c>1-\gamma \pi[\lambda(b, a b)+\lambda(b, b)]-\delta c
$$

or if

$$
z>-\pi\{r+\delta+\gamma[1-\lambda(a, a)]\}=z_{1}
$$

Then, any $z>z_{1}$ will prefer strategy $\{a, a b\}$ to $\{a, a\}$.

3. Strategy $\{b, a b\}$. Following the argument above we find the trade value of $a$ is

$$
\tau_{b, a b}(a)=v_{b, a b}(b)+[\lambda(a, a)+\lambda(a, a b)]\left[v_{b, a b}(a)-v_{b, a b}(b)\right]-\pi \lambda(b, b)
$$

while the trade value of possessing $b$ is

$$
\tau_{b, a b}(b)=v_{b, a b}(b)+\lambda(a, a b)\left[v_{b, a b}(a)-v_{b, a b}(b)\right]-\pi \lambda(a, a)
$$


The consumption value for $a$ satisfies

$$
\begin{aligned}
r v_{b, a b}(a)= & 1+\delta\left[v_{b, a b}(b)-v_{b, a b}(a)-c\right] \\
& +\gamma\left(v_{b, a b}(b)-v_{b, a b}(a)-[\lambda(a, a)+\lambda(a, a b)]\left[v_{b, a b}(b)-v_{b, a b}(a)\right]-\pi \lambda(b, b)\right)
\end{aligned}
$$

and the consumption value for $b$ satisfies

$$
r v_{b, a b}(b)=1+z-\delta c+\gamma\left[-\lambda(a, a b)\left[v_{b, a b}(b)-v_{b, a b}(a)\right]-\pi \lambda(a, a)\right]
$$

The implied difference is

$$
v_{b, a b}(b)-v_{b, a b}(a)=\frac{z+\gamma \pi[\lambda(b, b)-\lambda(a, a)]}{r+\delta+\gamma-\gamma \lambda(a, a)}
$$

We can now solve for the consumption value $v_{b, a b}(b)$ (for this strategy, the continuation value for an agent who gets the build shock is $\left.v_{b, a b}(b)-c\right)$.

$$
r v_{b, a b}(b)=1+z+\gamma\left[-\lambda(a, a b)\left[\frac{z+\gamma \pi[\lambda(b, b)-\lambda(a, a)]}{r+\delta+\gamma[1-\lambda(a, a)]}\right]-\pi \lambda(a, a)\right]-\delta c
$$

As before, we compare the continuation value for an agent who just received the build shock for strategies $\{b, a b\}$ and $\{a, a b\}$. The agent will prefer $\{b, a b\}$ to $\{a, a b\}$ if $v_{b, a b}(b) \geq v_{a, a b}(a) \Longleftrightarrow$

$$
\begin{aligned}
& 1+z+\gamma\left[-\lambda(a, a b)\left[\frac{z+\gamma \pi[\lambda(b, b)-\lambda(a, a)]}{r+\delta+\gamma[1-\lambda(a, a)]}\right]-\pi \lambda(a, a)\right]-\delta c \\
\geq & 1+\gamma\left\{\lambda(b, a b)\left[\frac{z+\gamma \pi[\lambda(b, b)-\lambda(a, a)]}{r+\delta+\gamma[1-\lambda(b, b)]}\right]-\pi \lambda(b, b)\right\}-\delta c
\end{aligned}
$$

This expression can be rewritten as

$$
(z+\gamma \pi[\lambda(b, b)-\lambda(a, a)])\left\{1-\frac{\gamma \lambda(a, a b)}{r+\delta+\gamma[1-\lambda(a, a)]}-\frac{\gamma \lambda(b, a b)}{r+\delta+\gamma[1-\lambda(b, b)]}\right\} \geq 0
$$

or

$$
(z+\gamma \pi[\lambda(b, b)-\lambda(a, a)]) \Omega \geq 0
$$

where

$$
\Omega \equiv 1-\frac{\gamma \lambda(a, a b)}{r+\delta+\gamma[1-\lambda(a, a)]}-\frac{\gamma \lambda(b, a b)}{r+\delta+\gamma[1-\lambda(b, b)]}
$$

We now show that $\Omega>0$. Without loss of generality assume that $\lambda(b, b) \geq \lambda(a, a)$. Then,

$$
\Omega \geq 1-\gamma\left[\frac{\lambda(b, a b)+\lambda(a, a b)}{r+\delta+\gamma[1-\lambda(b, b)]}\right]
$$

$\Omega>0$ if

$$
\begin{gathered}
1>\gamma\left[\frac{\lambda(b, a b)+\lambda(a, a b)}{r+\delta+\gamma[1-\lambda(b, b)]}\right] \\
r+\delta+\gamma[1-\lambda(b, b)-\lambda(b, a b)-\lambda(a, a b)]>0
\end{gathered}
$$

which is satisfied since $\lambda(b, a b)+\lambda(a, a b)+\lambda(a, a)+\lambda(b, b)=1$. This implies that $\Omega>0$. Because $\Omega>0$, agents will prefer $\{b, a b\}$ to $\{a, a b\}$ whenever

$$
z \geq \gamma \pi[\lambda(a, a)-\lambda(b, b)]=z_{2} .
$$

Any $z>z_{2}$ will prefer strategy $\{b, a b\}$ to $\{a, a b\}$. Furthermore, $z_{2}>z_{1}$ so these agents also prefer $\{b, a b\}$ to $\{a, a\}$. 
4. Strategy $\{b, b\}$. An agent who follows $\{b, b\}$ always rejects $a$ so $\tau_{b, b}(a)$ is irrelevant. The trade value $\tau_{b, b}(b)$ is

$$
\begin{aligned}
\tau_{b, b}(b) & =[\lambda(b, b)+\lambda(b, a b)] v_{b, b}(b)+[\lambda(a, a b)+\lambda(a, a)]\left[v_{b, b}(b)-\pi\right] \\
& =v_{b, b}(b)-\pi[\lambda(a, a b)+\lambda(a, a)]
\end{aligned}
$$

The consumption value is

$$
v_{b, b}(b)=\frac{1+z-\delta c-\gamma \pi[\lambda(a, a b)+\lambda(a, a)]}{r} .
$$

As before, consider an agent who receives the build shock. If he decides to follow $\{b, a b\}$ then his payoff is $v_{b, a b}(a)-c$ while if he follows $\{b, b\}$ his payoff is $v_{b, b}(a)-c$. The agent will thus prefer $\{b, b\}$ to $\{b, a b\}$ if $v_{b, b}(b)>v_{b, a b}(b) \Leftrightarrow$

$$
\begin{gathered}
1+z-\delta c-\gamma \pi[\lambda(a, a b)+\lambda(a, a)] \\
>1+z+\gamma\left[-\lambda(a, a b)\left[\frac{z+\gamma \pi[\lambda(b, b)-\lambda(a, a)]}{r+\delta+\gamma[1-\lambda(a, a)]}\right]-\pi \lambda(a, a)\right]-\delta c \\
z>\pi[r+\delta+\gamma[1-\lambda(b, b)]]=z_{3}
\end{gathered}
$$

Any $z>z_{3}$ will play strategy $\{b, b\}$ rather than $\{b, a b\}$.

Since $z_{1}<z_{2}<z_{3}$ any agent with $z<z_{1}$ prefers $\{a, a\}$ to all of the other strategies. To see this note that such an agent prefers $\{a, a\}$ to $\{a, a b\}$ by case one above. However, by case two, he also prefers $\{a, a b\}$ to $\{b, a b\}$ and by case three prefers $\{b, a b\}$ to $\{b, b\}$. Thus $\{a, a\}$ is the optimal strategy for this agent. Similar arguments imply that for $z_{1}<z<z_{2}$ the optimal strategy is $\{a, a b\}$; for any $z_{2}<z<z_{3}$ the optimal strategy is $\{b, a b\}$ and for $z>z_{3}$ the optimal strategy is $\{b, b\}$. This completes the proof.

Proposition 2 Given any distribution of types $F$, there exists at least one steady state equilibrium.

Proof. By Lemma $4 L_{a, a}$ and $L_{b, b}$ have at least one fixed point. Let $\lambda(a, a)$, and $\lambda(b, b)$ be fixed points of $L_{a, a}$ and $L_{b, b}$. We construct an equilibrium as follows:

Use $\lambda(a, a)$, and $\lambda(b, b)$ to construct $z_{1}, z_{2}$, and $z_{3}$ from the definitions in 1 . With $z_{1}, z_{2}$, and $z_{3}$ compute $\lambda(a, a), \lambda(b, b), \lambda(a, a b)$, and $\lambda(b, a b)$ in accordance with Lemma 3. By construction $\lambda(a, a)$, $\lambda(b, b), \lambda(a, a b)$, and $\lambda(b, a b)$ are equilibrium matching probabilities.

Proposition 3 If $F$ satisfies Assumption 1 then

1. The equilibrium is unique.

2. There is conformity whenever $\mu \neq 0$ and the market conforms to the mean taste (the market conforms on $a$ if $\mu<0$ and conforms on $b$ if $\mu>0$ ).

3. If $\mu \neq 0$, an increase in durability (lower $\delta$ ), patience (lower $r$ ), the liklihood of trade (higher $\gamma$ ) or the trade penalty (higher $\pi$ ) causes conformity to increase.

\section{Proof.}

1. By proposition $2, \lambda(a, a)$ is a fixed point of the mapping $L_{a, a}$,

$$
\lambda(a, a)=L_{a, a}(\lambda(a, a))=F(-\pi\{r+\delta+\gamma[1-\lambda(a, a)]\})
$$

By assumption $F$ has a density and thus we can calculate the derivative of $L_{a, a}$. This derivative is

$$
\frac{\partial L_{a, a}(\lambda(a, a))}{\partial \lambda(a, a)}=f\left(z_{1}\right) \pi \gamma>0
$$

The assumption guarantees that $f\left(z_{1}\right) \leq f(\mu)<\pi \gamma^{-1}$ which implies that the derivative satisfies $0<f\left(z_{1}\right) \pi \gamma<1$. Thus there can be at most one crossing. Similar arguments hold for $L_{b, b}$. Since existence of at least one equilibrium is guaranteed by proposition 2 , the equilibrium must be unique. 
2. Because $F$ is symmetric about its mean, if $\mu=0$ then the unique equilibrium must have $\lambda(a, a)=$ $\lambda(b, b)$ and $z_{2}=0$ (no conformity). Let $\lambda^{*}$ be the equilibrium $\lambda^{*}=\lambda(a, a)=\lambda(b, b)$ associated with $\mu=0$. Note that for any $l \geq \lambda^{*}$ we must have $L_{a, a}(l) \leq l$ (since the derivative of $L_{a, a}$ is less than 1 by part 1) and for any $l \leq \lambda^{*}$ we must have $L_{b, b}(l) \geq l$ by the same reasoning.

Consider $\mu>0$ (the argument for $\mu<0$ is identical). In this case, for any given $l \in[0,1]$,

$$
F(-\pi\{r+\delta+\gamma[1-l]\} ; \mu>0)<F(-\pi\{r+\delta+\gamma[1-l]\} ; \mu=0)
$$

and

$$
1-F(\pi\{r+\delta+\gamma[1-l]\} ; \mu>0)>1-F(\pi\{r+\delta+\gamma[1-l]\} ; \mu=0)
$$

Therefore,

$$
\begin{gathered}
\left.L_{a, a}(l)\right|_{\mu>0}<\left.L_{a, a}(l)\right|_{\mu=0} \leq l \\
\left.L_{b, b}(l)\right|_{\mu>0}>\left.L_{b, b}(l)\right|_{\mu=0} \geq l
\end{gathered}
$$

This implies that when $\mu>0, l \in\left[\lambda^{*}, 1\right]$ cannot be a fixed point of $L_{a, a}$ and $l \in\left[0, \lambda^{*}\right]$ cannot be a fixed point of $L_{b, b}$. Because the equilibrium is unique, we conclude that for $\mu>0$, the equilibrium satisfies $\lambda(b, b)>\lambda^{*}>\lambda(a, a)$. This implies that $z_{2}<0$ so consumers conform on $b$.

3. If $\mu>0$ then $1-F\left(z_{3}\right)=\lambda(b, b)>\lambda(a, a)=F\left(z_{1}\right)$ by part (2). This implies that $z_{2}>0$. Moreover, Lemma 7 implies $f\left(z_{3}\right) \geq f\left(z_{1}\right)$. Since $f\left(z_{1}\right) \leq f(\mu)<\pi \gamma^{-1}, \frac{\gamma \pi f\left(z_{1}\right)}{1-\pi \gamma f\left(z_{1}\right)}>0$ and $\frac{\gamma \pi f\left(z_{3}\right)}{1-\pi \gamma f\left(z_{3}\right)}>0$. The proof then follows by directly computing the derivatives $\frac{\partial z_{2}}{\partial \delta}, \frac{\partial z_{2}}{\partial r}, \frac{\partial z_{2}}{\partial \pi}$ and $\frac{\partial z_{2}}{\partial \gamma}$.

Proposition 4 For fixed $\lambda(a, a), \lambda(a, a b), \lambda(b, a b)$, and $\lambda(b, b)$, there exist two cutoffs $\rho \in\{-\infty\} \cup\left[z_{1}, 0\right]$ and $\eta \in\left[0, z_{3}\right] \cup\{+\infty\}$ such that

1. All agents with taste parameter $z \leq \rho$ choose to rent type a houses.

2. All agents with taste parameter $z \geq \eta$ choose to rent type $b$ houses.

3. All agents with taste parameter $z \in(\rho, \eta)$ remain in the owner-occupied market and follow policies prescribed in Proposition 1.

Proof. Let $v_{a, a}, v_{a, a b}, v_{b, a b}$ and $v_{b, b}$ be the values of following each strategy given that the agent is currently in the build stage. These values are defined in the proof of Proposition 1 and satisfy

$$
\begin{gathered}
r v_{a, a}(a)=1-\delta c-\gamma \pi[\lambda(b, a b)+\lambda(b, b)]-r c \\
r v_{a, a b}(a)=1+\gamma\left[\lambda(b, a b)\left[\frac{z+\gamma \pi[\lambda(b, b)-\lambda(a, a)]}{r+\delta+\gamma[1-\lambda(b, b)]}\right]-\pi \lambda(b, b)\right]-\delta c-r c \\
r v_{b, a b}(b)=1+z+\gamma\left[-\lambda(a, a b)\left[\frac{z+\gamma \pi[\lambda(b, b)-\lambda(a, a)]}{r+\delta+\gamma[1-\lambda(a, a)]}\right]-\pi \lambda(a, a)\right]-\delta c-r c \\
r v_{b, b}(b)=1+z-\delta c-\gamma \pi[\lambda(a, a b)+\lambda(a, a)]-r c
\end{gathered}
$$

An agent in the build stage can contemplate following one of the ownership policies (as above) or deviating to the rental market. Thus in the build stage he chooses the maximum of $\{R, V(a)-c, V(b)-c\}$ where $R$ is

$$
R=\max \left\{\frac{1-q}{r}, \frac{1+z-q}{r}\right\}
$$

Clearly these values depend on the agents type $z$. We thus write the values $v_{a, a}, v_{a, a b}, v_{b, a b}$ and $v_{b, b}$ as functions of $z$. Specifically, let $v_{a, a b}(z)$, and $v_{b, a b}(z)$ be the values of following strategy $\{a, a b\}$ and $\{b, a b\}$ respectively for an agent with taste parameter $z$ who is currently in the build stage. The slopes, $\frac{\partial v}{\partial z}$, of these values are increasing functions of $z$. Specifically, directly differentiating the expressions above gives:

$$
\frac{\partial v_{a, a}}{\partial z}=0<\frac{\partial v_{a, a b}}{\partial z}=\left(\frac{1}{r}\right)\left(\frac{\gamma \lambda(b, a b)}{r+\delta+\gamma[1-\lambda(b, b)]}\right)<\frac{\partial v_{b, a b}}{\partial z}=\left(\frac{1}{r}\right)\left(\frac{r+\delta+\gamma[\lambda(b, a b)+\lambda(b, b)]}{r+\delta+\gamma[1-\lambda(a, a)]}\right)<\frac{\partial v_{b, b}}{\partial z}=\frac{1}{r}
$$


The slopes of these values $\left(\frac{\partial v}{\partial z}\right)$ are increasing. ${ }^{1}$

Define $\rho_{a, a b}, \rho_{b, a b}$ implicitly as follows

$$
\begin{aligned}
& 1-q=r v_{a, a b}\left(\rho_{a, a b}\right) . \\
& 1-q=r v_{b, a b}\left(\rho_{b, a b}\right) .
\end{aligned}
$$

Let $\rho=\min \left\{\rho_{a, a b}, \rho_{b, a b}\right\}$. Note that no one with $z>0$ will choose to rent $a$ so the intersection of the $a$ rental line with $v_{b, b}$ is irrelevant. Define $\eta_{a, a b}$ and $\eta_{b, a b}$ implicitly as follows

$$
\begin{aligned}
& 1+\eta_{a, a b}-q=r v_{a, a b}\left(\eta_{a, a b}\right) . \\
& 1+\eta_{b, a b}-q=r v_{b, a b}\left(\eta_{b, a b}\right) .
\end{aligned}
$$

Let $\eta=\max \left\{\eta_{a, a b}, \eta_{b, a b}\right\}$.

If $\rho>\eta$ then everyone rents $(z>0$ rent $b$ and $z<0$ rent $a)$. In the other cases, $\rho<\eta$ and every $z \in[\rho, \eta]$ remains in the owner occupied market. If $z_{1}>\rho$ then no one will rent $a$. If $z_{1}<\rho$ then every $z<\rho$ rents $a$. Thus the number of people who rent $a$ is $F(\rho)$. If $z_{3}<\eta$ then no one will rent $b$. If $z_{3}>\eta$ then every $z>\eta$ rents $b$. Thus, the number of people who rent $b$ is $1-F(\eta)$.

Proposition 5 Let $f$ be symmetric about $\mu>0$, quasi-concave and single peaked with $f(\mu)<\frac{1}{\pi \gamma} \frac{1}{2}$ and assume that $\frac{1}{2} \gamma \pi<m c<\gamma \pi$. If the equilibrium requires an active rental market, then there exists an equilibrium in which only agents who prefer a houses rent. Moreover, in this equilibrium, the owner-occupied market has greater conformity than the equilibrium without the possibility of rent.

Proof. Since $\mu>0$, by Lemma 8 we have $\lambda^{0}(b, b)+\lambda^{0}(b, a b)>\frac{1}{2}>\lambda^{0}(a, a)+\lambda^{0}(a, a b)$ where we are using the superscript notation as described in the text. Consider agents who played strategy $(a, a)$ in the original equilibrium. Since $z_{1}<0$, these agents all strictly prefer type $a$ houses and thus would rent a type $a$ house if they left the owner-occupied market. These agents will strictly prefer renting if

$$
\frac{1-q}{r}>v_{a, a}(a)=\frac{1-\delta c-\gamma \pi\left[\lambda^{0}(b, a b)+\lambda^{0}(b, b)\right]}{r}-c
$$

Using the definition of $q$ we see that these agents will rent if

$$
(m) c<\gamma \pi\left[\lambda^{0}(b, a b)+\lambda^{0}(b, b)\right]
$$

The same reasoning implies that agents who played $(b, b)$ in the original equilibrium will prefer renting if

$$
(m) c<\gamma \pi\left[\lambda^{0}(a, a b)+\lambda^{0}(a, a)\right] .
$$

Since $\lambda^{0}(b, b)+\lambda^{0}(b, a b)>\frac{1}{2}>\lambda^{0}(a, a)+\lambda^{0}(a, a b)$, and because by assumption $(m) c>\frac{1}{2} \gamma \pi$, either the $(a, a)$ agents prefer to rent at the original equilibrium or the original equilibrium is still a valid equilibrium with an inactive rental market.

Suppose that $(m) c<\gamma \pi\left[\lambda^{0}(b, a b)+\lambda^{0}(b, b)\right]$ so that the $(a, a)$ types prefer renting faced with the initial equilibrium in the owner-occupied market. In this case, the original equilibrium will not survive (the $(a, a)$ types will deviate).

We construct a mapping $\Phi:[0, F(0)] \rightarrow[0, F(0)]$ as follows: Take any $\phi \in[0, F(0)]$, and let $\zeta=F^{-1}(\phi)$ $(\zeta$ solves $\phi=F(\zeta))$. Construct $\tilde{F}$ and $f$ as follows:

$$
\tilde{F}(z)=\left\{\begin{array}{cc}
0 & \text { for } z \leq \zeta \\
\frac{F(z)-\phi}{1-\phi} & \text { for } z>\zeta
\end{array} \text { and } \tilde{f}(z)=\left\{\begin{array}{cc}
0 & \text { for } z \leq \zeta \\
\frac{f(z)}{1-\phi} & \text { for } z>\zeta
\end{array} .\right.\right.
$$

${ }^{1}$ Note that $\frac{\partial v_{b, a b}}{\partial z}>\frac{\partial v_{a, a b}}{\partial z}$. To see this, recall the definition of $\Omega$ from the proof of Proposition 1

$$
0<\Omega \equiv 1-\frac{\gamma \lambda(a, a b)}{r+\delta+\gamma[1-\lambda(a, a)]}-\frac{\gamma \lambda(b, a b)}{r+\delta+\gamma[1-\lambda(b, b)]}=r\left[\frac{\partial v_{b, a b}}{\partial z}-\frac{\partial v_{a, a b}}{\partial z}\right] .
$$

The inequality follows from the proof of proposition 1. 
It is easy to show that $\tilde{F}(z)-F(z)=-\frac{\phi}{1-\phi}[1-F(z)]$ so that $\tilde{F}(z) \leq F(z)$ (and thus $\left.1-\tilde{F}(z)>1-F(z)\right)$. The associated density function has $\tilde{f}(z)=\frac{f(z)}{1-\phi}<\frac{f(0)}{1-\phi}<2 f(\mu)<\frac{1}{\pi \gamma}$ where the last inequality follows from our assumptions on $F$. As a result, $\tilde{f}$ satisfies the requirements for a unique equilibrium in the owner-occupied market. Let

$$
L_{a, a}\left(\lambda^{1}(a, a)\right)=\tilde{F}\left(-\pi\left\{r+\delta+\gamma\left[1-\lambda^{1}(a, a)\right]\right\}\right) \in[0,1]
$$

and

$$
L_{b, b}\left(\lambda^{1}(b, b)\right)=1-\tilde{F}\left(\pi\left\{r+\delta+\gamma\left[1-\lambda^{1}(b, b)\right]\right\}\right) \in[0,1] .
$$

be the fixed point mappings for the owner-occupied market with the distribution $\tilde{F}$. Since $\tilde{f}<\frac{1}{\pi \gamma}$ there is a unique equilibrium. Let $\lambda^{1}(a, a), \lambda^{1}(a, a b), \lambda^{1}(b, b), \lambda^{1}(b, a b)$ be the unique equilibrium set of trading probabilities. Because $\tilde{F}(z) \leq F(z)$ and $1-\tilde{F}(z) \geq 1-F(z)$, it must be the case that there is greater conformity in the new equilibrium than in the original equilibrium without the rental market. That is, $\lambda^{1}(a, a)<\lambda^{0}(a, a)$ and $\left.\lambda^{1}(b, b)>\lambda^{0}(b, b)\right)$. Moreover, conformity grows with increases in $\phi$. Define $\rho$ as above. We set $\phi^{\prime}=\tilde{F}(\rho)$.

Note that if the original equilibrium is no longer viable then $\Phi(0)>0$. Also, if $\phi=F(0)$ then everyone remaining in the owner-occupied market has $z>0$. As a result, in equilibrium, everyone produces and trades type $b$ houses. In this case $\lambda^{1}(a, a b)=\lambda^{1}(a, a)=0$ and thus, $\rho_{b, a b}=-(m) c$. Since $\rho=\min \left\{\rho_{a, a b}, \rho_{b, a b}\right\}$ we have $\Phi(F(0))=F(\rho)<F(0)$.

Thus, we have a mapping $\Phi$ on $[0, F(0)]$ which satisfies $\Phi(0)>0$ and $\Phi(F(0))<F(0)$. By the intermediate value theorem (or by Brouwer's Fixed Point Theorem) we must have a fixed point in the interval. Since $\lambda^{1}(a, a)<\lambda^{0}(a, a)$ and $\lambda^{1}(b, b)>\lambda^{0}(b, b)$ for any $\phi \in[0, F(0)]$, we conclude that in the equilibrium with an active rental market there is even greater conformity than when there was no possibility of renting. 
FIGURE 1: UNIQUE EQUILIBRIA WITH A UNIFORM DISTRIBUTION

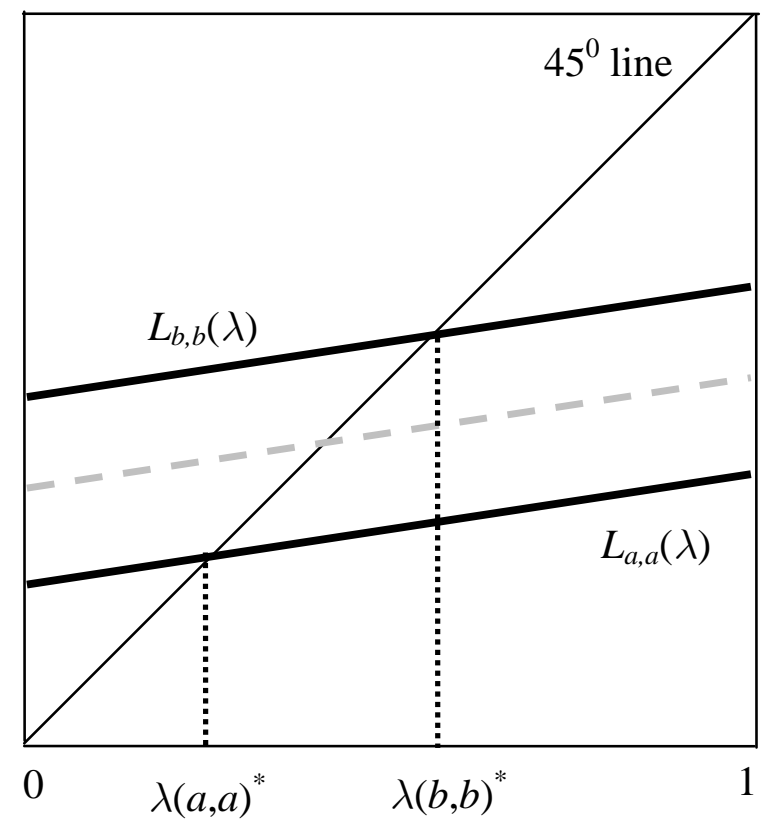

Figure 2: Multiple EQUiLIBRIA WITH A SYMMETRIC DistribUtion

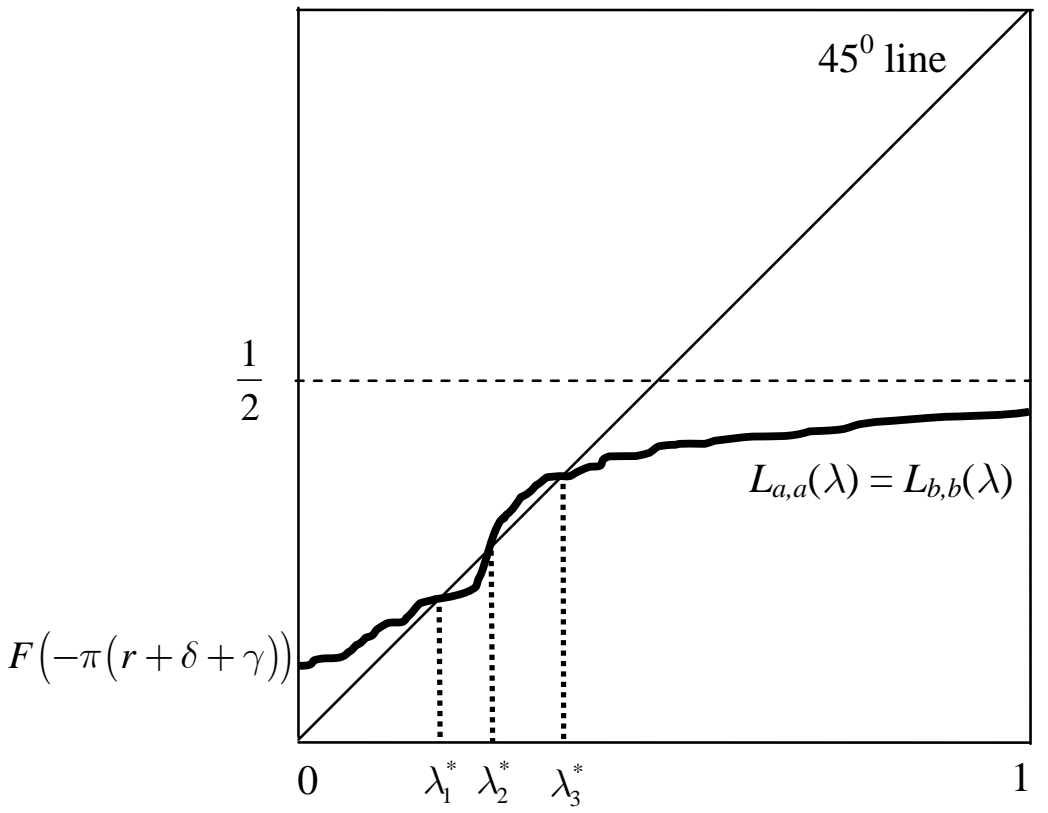




\section{FIGURE 3: WELFARE AS A FUNCTION OF $z_{2}$}

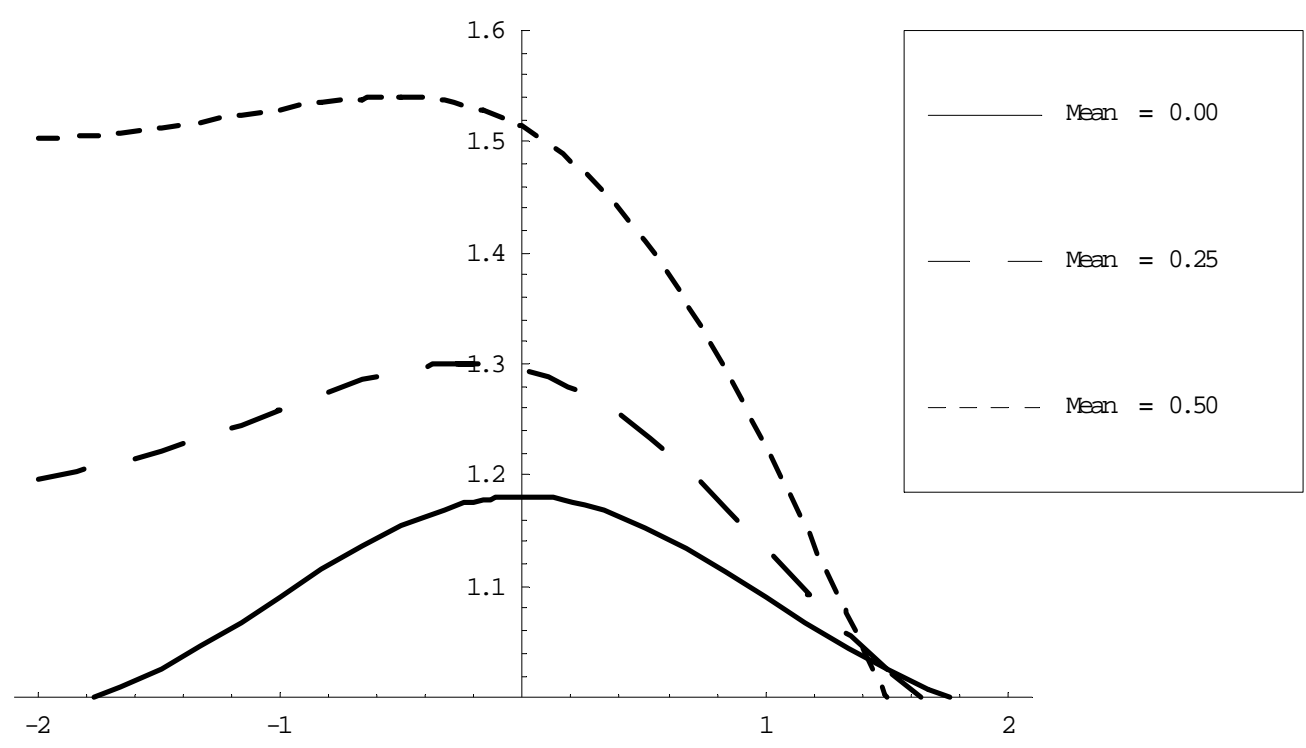

The figure plots the flow of welfare for the model when $F(z)$ is distributed normally. Three means $(0, .25$ and .5) are considered. Each distribution has the same standard deviation (1).

Figure 4: EqUiLIBRIUM AND Optimal $z_{1}, z_{2}, z_{3}$ FOR DiffERENT MEANS.

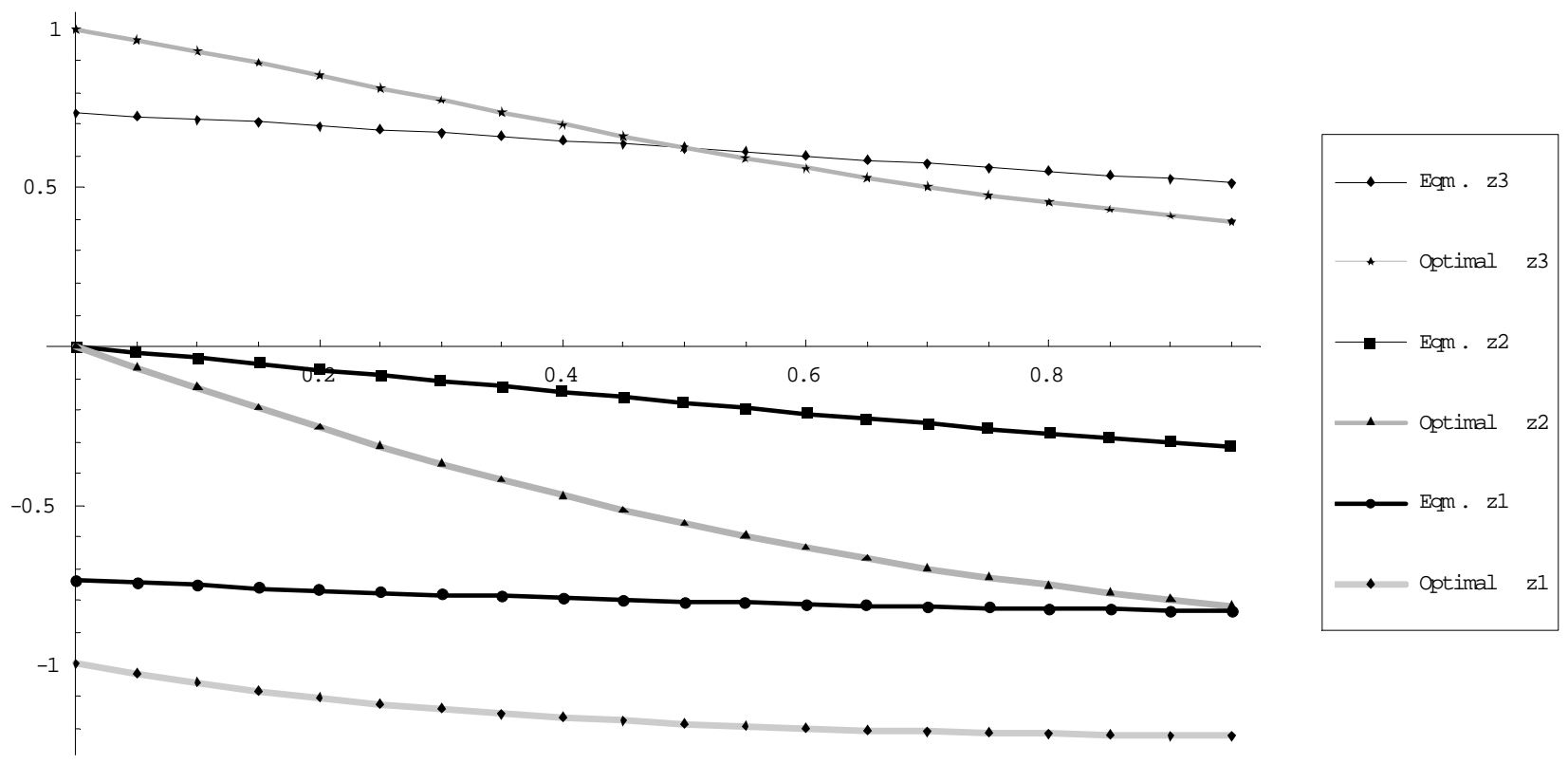

The figure shows the equilibrium cutoffs (the solid dark lines) and the optimal cutoffs (the light shaded lines) for different means of $F(z)$. In each case, $F$ is normal with a unit variance. The cutoffs are plotted on the vertical axis while the mean is on the horizontal axis. 